\title{
Distribution Route Optimization for Electric Vehicles in Urban Cold Chain Logistics for Fresh Products under Time-Varying Traffic Conditions
}

\author{
Zhixue Zhao ${ }^{1}{ }^{1,2}$ Xiamiao Li, ${ }^{1}$ and Xiancheng Zhou $^{2}$ \\ ${ }^{1}$ School of Traffic \& Transportation Engineering, Central South University, Changsha 410083, China \\ ${ }^{2}$ Key Laboratory of Hunan Province for Mobile Business Intelligence, Hunan University of Technology and Business, \\ Changsha 410205, China \\ Correspondence should be addressed to Zhixue Zhao; zhaozhixue90@126.com
}

Received 19 May 2020; Revised 13 August 2020; Accepted 23 September 2020; Published 8 October 2020

Academic Editor: Gaetano Zizzo

Copyright (c) 2020 Zhixue Zhao et al. This is an open access article distributed under the Creative Commons Attribution License, which permits unrestricted use, distribution, and reproduction in any medium, provided the original work is properly cited.

Electric vehicles (EVs) have been widely used in urban cold chain logistic distribution and transportation of fresh products. In this paper, an electric vehicle routing problem (EVRP) model under time-varying traffic conditions is designed for planning the itinerary for fresh products in the urban cold chain. The object of the EVRP model is to minimize the total cost of logistic distribution that includes economic cost and fresh value loss cost. To reflect the real situation, the EVRP model considers several influencing factors, including time-varying road network traffic, road type, client's time-window requirement, freshness of fresh products, and en route queuing for charging. Furthermore, to address the EVRP, an improved adaptive ant colony algorithm is designed. Simulation test results show that the proposed method can allow EVs to effectively avoid traffic congestion during the distribution process, reduce the total distribution cost, and improve the performance of the cold chain logistic distribution process for fresh products.

\section{Introduction}

With the increase of greenhouse effect, clean energy is the energy of the future for traffic development. With government support, electric vehicles (EVs) are becoming an industry trend as people seek ways to reduce emissions to protect the environment. EVs, an ideal means of transportation to replace conventional fuel vehicles, are energy-efficient, green, and environmentally friendly. Therefore, EVs have received extensive attention to combat the deteriorating air pollution and global climate change. Many countries have set policies and developed several projects to improve the penetration of EVs in their daily lives. By 2017, EVs (including pure electric vehicles and plug-in hybrid vehicles) exceeded 3 million around the world, an increase of 57\% compared with 2016. Data show that the number of pure electric vehicles in China rose to 3.1 million in 2019, which is the highest number of EVs compared with the EV numbers for a few years ago, accounting for about $40 \%$ of the global EV ownership. It is foreseen that the number of EVs will break through 200 million in 2030 [1]. For instance, Beijing released the "Beijing New Energy Logistics and Distribution Vehicle Priority Implementation Plan" in 2019: by the end of 2020, light electric logistic vehicles under 4.5 tons will account for 90 percent of the total freight vehicles. While they have advantages (e.g., low energy consumption and zero pollution), EVs also have disadvantages, namely, long charging time, limited battery capacity, and relatively low mileage on a single charge $[2,3]$. With the increasing popularization of EVs, how to scientifically dispatch EVs and optimize logistic distribution routes under the constraints of EV technology to achieve economic and environmental protection goals has continually been a focus of researchers.

The EVRP is an extension of vehicles routing problem (VRP), but there are obvious differences between them. (1) Different transport means result in different energy consumption functions. Compared with traditional vehicles, EVs generally have a range constraint of about $100-200 \mathrm{~km}$. Due to the characteristics of urban time-varying networks, road congestion, and customer point service requirements, the EV range may be reduced. To meet distribution needs, 
EVs may need to be recharged in transit. (2) The EVRP needs to consider the charging strategy of EVs in the routing planning process [4]. (3) If EVs need en route fast charging in the EVRP, the selection of social charging stations, queuing, charging time, and charging efficiency have an important impact on path planning. Therefore, the EVRP is more complex compared with traditional VRP.

Currently, the EVRP is a focal area of research. Beltran et al. [5] proposed using EVs in urban transportation and established an optimization model with the minimization of the transportation distance as an objective. Erdoan and MillerHooks [6] were the first to put forward the EVRP. In view of the problems of EVs (i.e., low mileage on a single charge and a lack of charging facilities), they studied an EVRP with time windows that accounted for charging strategies. In addition, they established a mixed integer model with the minimization of the travel distance as an objective and solved it using an improved saving algorithm and a clustering algorithm. Schneider et al. [7] studied the last-kilometer logistics of EVs and took into account the effects of client's time window, vehicle load and single-charge mileage constraints, and en route charging. They solved the problem using a variable neighborhood search and tabu search-combined heuristic algorithm. Goeke and Schneider [8] studied an EV power consumption model based on relevant factors, such as vehicle speed, vehicle load, and road gradient, and established an EVRP model for driving and energy consumption costs by considering charging strategies. Yang et al. [9] considered the time-of-use (ToU) electricity price when optimizing and solving the EVRP and designed an EV route planning and charging strategy selection model under the ToU electricity price. They also designed a genetic algorithm for solving this model. Desaulniers et al. [10] took into consideration four charging strategies when optimizing an EVRP with time windows. They obtained an optimum charging method by optimizing the four charging strategies under a client timewindow constraint. Jie et al. [11] and Hiermann et al. [12] examined a combination of multiple vehicle types and the logistic distribution route problem of EVs. Jie et al. [11] distinguished various vehicle types based on factors such as battery capacity, power consumption rate per unit, and vehicle load and designed a branch-and-price algorithm for finding an optimal solution to the problem. Hiermann et al. [12] rationally configured the fleet structure for distribution based on the differences between EV types in terms of energy consumption and load. To better conform to reality, Li et al. [13] planned mixed fleets of conventional vehicles and EVs for enterprises and thereby provided decision-making support to the government for choosing locations of charging facilities and to enterprises for logistic operation management.

Cold chain logistics for fresh food products is also a focus of research in China and elsewhere. Tiwari and Chang [14] optimized the route of vehicles in cold chain logistics for fresh products with the minimization of the travel distance and carbon emissions as objectives. Kumar et al. [15] established a multiobjective (total cost and total emissions) cold chain transportation route problem. Rabbani et al. [16], Amorim and Almada-Lobo [17], and $\mathrm{Wu}$ et al. [18] introduced the freshness of fresh products into cold chain distribution route optimization for fresh products. Zhang and Liang [19] established a cold chain vehicle route optimization model with the minimization of the total cost of cold chain logistic network construction and transportation as an objective. Devapriya et al. [20] established a coordinated production and distribution optimization model for perishable products with the minimization of the total distribution cost as an objective. They also designed a mixed heuristic algorithm to solve the model. Li et al. [21] investigated a new integrated planning problem for intelligent food logistic systems. They formulated a biobjective mixed integer linear programming model that considers the two objectives: minimizing total production, inventory, and transportation cost and maximizing average food quality.

The VRP is a typical NP- (nondeterministic polynomial-) hard problem. As the number of nodes increases rapidly, an exact algorithm is rather difficult to employ to solve largescale VRP. Currently, there are many VRP optimization algorithms, such as genetic algorithm [22], local search algorithm [23], tabu search algorithm (TSA) [24], simulated annealing search algorithm [25], and ant colony algorithm (ACA). According to the characteristics of the EVRP model in this paper, the ant colony algorithm is designed to solve the problem. Ant colony algorithm is a bionic random search algorithm originated from nature. Many scholars at home and abroad have applied it to various fields of life. It has made more outstanding achievements in combinatorial optimization, such as traveling salesman problem [26], scheduling problem [27], robot control system problem [28], and personalized trip planning problem [29]. ACA has the characteristics of distributed computing, no central control, and indirect communication between individuals, so it is easy to combine with other algorithms. For example, ACA combines with BP neural networks [30], genetic algorithm [31], etc. VRP is a complex discrete-time system, and many literature studies show that ACA has a broad development prospect in the field of VRP. Chen et al. [32] studied the optimization of the transportation routing problem for fresh food by an improved ant colony algorithm based on tabu search. Tang et al. [33] studied the low-carbon vehicle routing problem model; they designed an improved ant colony algorithm for solving this model, and chaotic disturbance mechanism was introduced to update the ant pheromones on the path. Fang and $\mathrm{Ai}$ [34] studied the hybrid ant colony algorithm to solve the cold chain logistic distribution optimization model.

A review of the literature published by researchers in China and elsewhere finds that in-depth research can still be conducted in the following several areas. (1) The available studies have mostly independently investigated the EVRP and the cold chain distribution route optimization problem but less frequently considered the optimization of urban cold chain distribution with EVs. (2) Studies on the effects of the external environment on the distribution with EVs mostly have considered that the vehicle speed is constant and there is only one road network type, overlooking the impact of dynamic traffic in a road network on the actual distribution process with EVs. (3) The available studies have mostly considered the biggest continue voyage course, but in the process of electric vehicle distribution, when and where to quickly 
charge is also a key issue. (4) When examining en route EV charging, most studies have only considered the charging time but overlooked the queuing time at the charging station. All of the above are the primary motivations of this research.

The main contributions of this paper can be summarized as follows: (1) According to the characteristics of fast charging of EVs in social charging stations, the queuing and charging process model of EVs is employed. (2) An improved adaptive ant colony algorithm is designed to solve the distribution optimization model with time windows for EVs in cold chain logistics for fresh products. (3) The charging station placement strategy based on the minimum power consumption principle is designed to solve the key problem of when and where to fast charge in the distribution process. (4) Based on the perspective of considering the economic cost of distribution and the cost of fresh value loss, the EVRP for urban fresh distribution under the time-varying road network is studied.

The remainder of this paper is organized as follows. In Section 2, we describe the mathematical model and the cost analysis of EVs in cold chain logistics. In Section 3, the improved ant colony algorithm is proposed with the entire design process of the algorithm. In Section 4, experiments with the Solomon benchmark are performed to analyze the impact of different types of cases and optimization objectives on the performance of the algorithm and compared with other excellent algorithms. Finally, Section 5 gives an overall conclusion.

\section{Description and Modeling of the Problem}

2.1. Description of the Problem and Basic Assumptions. The problem of distribution route optimization for EVs in cold chain logistics for fresh products under time-varying urban traffic conditions can be described as follows. A certain distribution center distributes fresh agricultural products to end clients via EVs. The EVs leave the distribution center, complete all the distribution tasks, and then return to the distribution center. The location, time window, and demand at each client point are known. The time-varying urban road network traffic information can be obtained from traffic control authorities. During the distribution process, the EVs can be charged at public charging stations as needed. Under these conditions, a distribution plan is formulated with the minimization of the total distribution cost as an objective. To clarify the scope of application of the study, the following assumptions are made: (1) There is only one distribution center in the distribution network. This center has an ample supply of EVs of the same type. (2) Each EV leaves the distribution center on a full charge and immediately returns after completing the tasks. (3) The demand at each client point is less than the capacity of each EV. There is also a service time-window requirement. An EV can reach a client point to provide service at an earlier or later time but then will pay a certain penalty. (4) Vehicle speeds in all the sections of the road network are updated every $10 \mathrm{~min}$. Considering the time-varying nature of network traffic, road sections are classified based on the areas where two arbitrary nodes are located. The road sections differ in time-varying characteristics. (5) When its remaining battery capacity cannot meet the distribution need, an EV will go to a charging station for a fast charge. (6) Each EV has a fixed maximum load capacity. In addition, one and only one EV serves each client point. (7) The locations of the public charging stations are known. The public charging stations have the same relevant charging parameters. The queuing time for charging is uncertain and depends on the time of day and the area.

2.2. Symbolic Parameters. $\mathbf{N}$ is the set of customer points; 0 represents the distribution center; $\mathbf{F}$ is the set of charging stations; $\mathbf{V}$ represents the set of all nodes in a logistics network, $\mathbf{V}=\mathbf{N} \cup \mathbf{F} \cup\{0\}$. $\mathbf{T}$ is the set of the time period throughout the day, $\mathbf{T}=\left\{T_{1}, T_{2}, \ldots, T_{P}\right\} ; P$ is the total number of time periods. $\mathbf{R}$ is the set of the road section in the road network, $\mathbf{R}=\left\{R_{1}, R_{2}, \ldots, R_{H}\right\} ; H$ is the number of road section types. $\mathbf{A}$ is the set of area, $\mathbf{A}=\left\{A_{1}, A_{2}, \ldots, A_{G}\right\} ; G$ is the number of the area types. Other descriptions about symbols are explained in Table 1.

\subsection{Elements and Variable Analysis of Distribution with EVS}

2.3.1. Analysis of Charging Needs and Power Consumption Performance. Currently, there are three modes of charging an $\mathrm{EV}$, namely, battery replacement, slow charging, and fast charging. Considering the high timeliness requirements of urban cold chain distribution of fresh products, fast charging in the delivery process is considered in this study. Time requirements are not considered during the slow-charging process. To overcome its low single-charge mileage problem during the distribution process and improve its distribution efficiency, an EV needs to undergo a fast charge at a public charging station. In addition, the relatively long queuing and charging process is an important component of distribution with EVs. See elsewhere [35] for a discussion of the queuing process. The queuing and charging process of an EV at a public charging station conforms to the $\mathrm{M} / \mathrm{G} / \mathrm{s}$ standard multiserver queuing system model ( $M$ means that the time interval for an EV to reach a charging station follows the exponential distribution; $G$ means that the charging time of an EV follows the normal distribution; there are a total of $s$ servers). Let $\rho_{i}$ be the utilization ratio of charging equipment in the charging station $i$ $\left(\rho_{i}=\left(\lambda_{i} / s_{i} \mu_{i}\right)\right)$. According to the classical little equation, the average queuing time for the $k^{\text {th }} \mathrm{EV}$ at charging station $i$ is

$$
t_{i k}^{Q}=\frac{\left(s_{i} \rho_{i}\right)^{s_{i}} \rho_{i}}{\lambda_{i} s_{i} !\left(1-\rho_{i}\right)^{2} \omega_{A_{i}}} \cdot\left(\sum_{n=0}^{s_{i}-1} \frac{\left(s_{i} \rho_{i}\right)^{n}}{n !}+\frac{\left(s_{i} \rho_{i}\right)^{s_{i}}}{s_{i} !\left(1-\rho_{i}\right)}\right)^{-1} y_{i k} .
$$

During the fast-charging process, the charging time for the $k^{\text {th }} \mathrm{EV}$ at charging station $i$ is as follows:

$$
t_{i k}^{C}=\frac{E_{\max }-E_{i k}^{L}}{r_{c}} y_{i k} \text {. }
$$

The energy consumption of electric vehicles is not only related to the nature of the vehicle but also related to the load and speed. In addition, part of the output power of the electric refrigerated vehicle is converted into mechanical power under certain transmission efficiency, and the parameters are shown in Table 2. When an EV whose load is $Q_{v}$ 
TABLE 1: Symbols and descriptions.

\begin{tabular}{|c|c|}
\hline Symbols & Descriptions \\
\hline $\mathbf{K}$ & The set of distribution vehicles, $\mathbf{K}=\{0,1,2, \ldots, k\}$ \\
\hline$Q$ & The vehicle maximum capacity $(\mathrm{kg})$ \\
\hline$E_{\max }$ & The maximum battery capacity of the EV $(\mathrm{kWh})$ \\
\hline$E_{\text {low }}$ & The minimum battery power level for the vehicle to drive normally $(\mathrm{kWh})$ \\
\hline$q_{i}$ & The demand of customer point $i, q_{i}<Q(\mathrm{~kg})$ \\
\hline$t_{i}^{S}$ & The unloading service time of customer point $i(\mathrm{~min})$ \\
\hline$\left[e_{i}, l_{i}\right]$ & The time window of customer point $i(\mathrm{~min})$ \\
\hline & The driving distance of the vehicle from node $i$ to node $j(\mathrm{~km}), i, j \in \mathbf{V}$ \\
\hline$t_{i j k}$ & The travel time of the $k^{\text {th }}$ vehicle on the road $(i, j)(\min ), k \in \mathbf{K}, i, j \in \mathbf{V}$ \\
\hline$t_{i k}^{A}$ & The time at which the $k^{\text {th }} \mathrm{EV}$ arrives at point $i(\min ), k \in \mathbf{K}, i \in \mathbf{V}$ \\
\hline$t_{i k}^{L}$ & The time at which the $k^{\text {th }}$ EV leaves point $i(\min ), k \in \mathbf{K}, i \in \mathbf{V}$ \\
\hline$E_{i k}^{A}$ & The remaining battery power level at the time of arriving at point $i(\mathrm{kWh}), k \in \mathbf{K}, i \in \mathbf{V}$ \\
\hline$E_{i k}^{L}$ & The remaining battery power level at the time of leaving point $i(\mathrm{kWh}), k \in \mathbf{K}, i \in \mathbf{V}$ \\
\hline$E_{i j k}$ & The power consumption for which the vehicle travels on the road $(i, j)(\mathrm{kWh}), k \in \mathbf{K}, i, j \in \mathbf{V},(i, j) \in \mathbf{R}$ \\
\hline$Q_{i k}^{A}$ & The load of the $k^{\text {th }} \mathrm{EV}$ at the time of arriving at point $i(\mathrm{kWh}), k \in \mathbf{K}, i \in \mathbf{V}$ \\
\hline$Q_{i k}^{L}$ & The load of the $k^{\text {th }} \mathrm{EV}$ at the time of leaving point $i(\mathrm{kWh}), k \in \mathbf{K}, i \in \mathbf{V}$ \\
\hline$P_{1}$ & The fixed cost of per EV (yuan) \\
\hline$P_{2}$ & The labor and rent cost of EV per unit time (yuan/min) \\
\hline$P_{3}$ & The unit electricity consumption (yuan/kWh) \\
\hline$P_{4}$ & The unit electricity price of fast charging (yuan $/ \mathrm{kWh})$ \\
\hline$P_{5}$ & The refrigeration costs which generate during transportation process of unit time (yuan/h) \\
\hline$P_{6}$ & The refrigeration costs which generate during unloading process of unit time (yuan/h) \\
\hline$P_{7}$ & The price of the unit commodity (yuan $/ \mathrm{kg}$ ) \\
\hline$P_{8}$ & The cost of waiting for the unit time if refrigerated EV arrives at customer node in advance (yuan/min) \\
\hline$P_{9}$ & The cost of punishing for the unit time if refrigerated EV is late to the customer node (yuan/min) \\
\hline$\lambda_{i}$ & The number of EVs that reach the charging station $i$ (vehicles/h) \\
\hline$\mu_{i}$ & The number of EVs that have completed charging per unit time in the charging station $i$ (vehicles/h) \\
\hline$s_{i}$ & The number of charging piles in the charging station $i$ \\
\hline$\partial_{1}$ & The freshness decay coefficient during the transportation process \\
\hline$\partial_{2}$ & The freshness decay coefficient during the unloading process \\
\hline$t_{i k}^{Q}$ & The average queuing time for EV $k^{\text {th }}$ at charging station $i(\mathrm{~min})$ \\
\hline$\omega_{A_{i}}$ & The area factor for charging demand which is introduced to differentiate the charging demand between different areas, $A_{i} \in \mathbf{A}$ \\
\hline$t_{i k}^{C^{i}}$ & The charging time for $k^{\text {th }} \mathrm{EV}$ at charging station $i$ (min) \\
\hline$r_{c}$ & The charging speed $(\mathrm{kWh} / \mathrm{min})$ of the public charging station \\
\hline$C N_{\max }$ & The maximum number of charging cycles \\
\hline$C E$ & The cost of a battery (yuan) \\
\hline$x_{i j k}$ & The binary variable that is 1 when the $k^{\text {th }} \mathrm{EV}$ is driving on road $(i, j)$ and 0 otherwise \\
\hline$y_{i k}$ & The binary variable that is 1 when the $k^{\text {th }} \mathrm{EV}$ is servicing customer point $i$ and 0 otherwise \\
\hline$Z_{i k}$ & The binary variable that is 1 when the $k^{\text {th }} \mathrm{EV}$ is charging at point $i$ and 0 otherwise \\
\hline
\end{tabular}

runs on a flat road at a speed of $v$, the running power $P\left(Q_{V}, v\right)$ is expressed as follows [36]:

$$
P\left(Q_{v}, v\right)=\frac{\left(\left(m+Q_{v}\right) \cdot g \cdot f \cdot v+\left(C_{d} \cdot W_{a} \cdot v^{3} / 21.15\right)\right)}{3600 \eta} .
$$

\subsubsection{Correlation Analysis of the Time-Varying Nature of a} Road Network. In a time-varying network, vehicle speeds vary with the time period. Within the same time period, vehicle speeds may also vary with the road section. Therefore, travel time is difficult to be calculated and needs to be rationally dealt with. Based on the previously proposed cross-time period travel time calculation methods $[37,38]$, the real-time speed of a vehicle within a sufficiently short time is used as its fixed speed within the time period. To reflect the variation in road sections where vehicles travel, it is also necessary to take into consideration the types of road sections. On this basis, the vehicle speed in the current road section is determined at the current moment. The following shows a step function for the speed at various times on an arbitrary road section:

$$
v_{i j}(t)=\left\{\begin{array}{cc}
v_{i j}^{1}, & t \in T_{1}, \\
v_{i j}^{2}, & t \in T_{2}, \quad(i, j) \in \mathbf{R}, \\
\ldots & \\
v_{i j}^{P}, & t \in T_{P},
\end{array}\right.
$$

where the velocity value does not change in the same period of time in the road $(i, j)$. A number of factors, including road 
TABLE 2: Parameters and descriptions about the EV consumption function.

\begin{tabular}{lc}
\hline Parameters & Descriptions \\
\hline$Q_{v}$ & The current load of EV $(\mathrm{kg})$ \\
$v$ & The current speed of EV $(\mathrm{km} / \mathrm{h})$ \\
$M$ & The bare vehicle weight $(\mathrm{kg})$ \\
$g$ & The acceleration of gravity $\left(\mathrm{m} / \mathrm{s}^{2}\right)$ \\
$\eta$ & The mechanical efficiency of the transmission system \\
$F$ & The rolling resistance coefficient \\
$C_{d}$ & The air resistance coefficient \\
$W_{a}$ & The windward area of the vehicle $\left(\mathrm{m}^{2}\right)$ \\
\hline
\end{tabular}

type, travel time period, and time-varying vehicle speed, are analyzed. The whole-day distribution time is evenly divided into multiple time periods. In a time-varying network, vehicle travel speeds are updated once every time period. Here, the traveling of a vehicle on a specific road section is used as an example. The travel time calculation process for the vehicle in the time-varying network is analyzed as follows:

(1) Determining the road type $r$ based on the areas where the points $i$ and $j$ are located, and, on this basis, the vehicle speed during the corresponding time period is determined. Go to (2).

(2) The distance traveled by the EV when it travels at the corresponding speed in the remaining time that is in the current period, plus the distance that the EV has already traveled, is calculated, and whether the total distance obtained exceeds the distance of $\operatorname{road}(i, j)$ is determined.

(3) If the total distance exceeds the distance of road $(i, j)$, the travel time is the sum of the travel time experienced by the EV before entering the current time period and in the current time period. Where the driving time of the $\mathrm{EV}$ in the current period is obtained as follows: the distance of road $(i, j)$ minus the distance previously experienced divided by the driving speed corresponding to the current period.

(4) If the total distance does not exceed the distance of road $(i, j)$, the EV will continue to drive in the next period. Go to (2) and repeat.

The pseudocode for calculating the traveling time $t_{i j k}$ in the time-varying traffic network is detailed in Algorithm 1, and the corresponding parameters and descriptions are shown in Table 3.

\subsubsection{Cost Analysis of EVS in Cold Chain Logistics.}

Based on the actual overall profit of fresh-food cold chain distribution enterprises, the minimization of the total distribution cost (including economic cost of distribution and fresh value loss cost, where the economic cost of distribution consists of the fixed cost, time cost, energy consumption cost, charging cost, refrigeration cost, and penalty cost) is set as the optimization objective. On this basis, a route optimization model for EVs in cold chain logistic distribution of fresh products is established.

$$
\begin{aligned}
& \text { (1) Compute_time }\left(d_{i j}, t_{i k}^{L}\right) \text { : } \\
& \text { (2) Set } t l_{k}=t_{i k}^{L}, d=d_{i j}, t_{i j k}=t l_{k}+\left(d / v_{i j}^{B}\right) \text {. } \\
& \text { (3) While }\left(t_{i j k}>t_{B+1}\right) \\
& \text { (4) } \quad d=d-v_{i j}^{B}\left(t_{B+1}-t l_{k}\right) \\
& \text { (5) } \quad t l_{k}=t_{B+1} ; \\
& \text { (6) } B=B+1 \\
& \text { (7) } t_{i j k}=\text { Compute_time }\left(d, t l_{k}\right) \\
& \text { (8) End while } \\
& \text { (9) Return } t_{i j k}
\end{aligned}
$$

Algorithm 1: Calculation of the traveling time $t_{i j k}$ in the timevarying traffic network.

(1). Fixed Cost of EVS. The fixed cost of EVs $\left(C_{1}\right)$ is primarily their front-end cost. This cost is only related to the number of refrigerated EVs that are used:

$$
C_{1}=P_{1} \cdot \sum_{k \in \mathbf{K}} \sum_{j \in \mathbf{V}} x_{0 j k}
$$

(2). Travel Cost of EVS. This cost $\left(C_{2}\right)$ is primarily the product of the cost per unit time and the total running time. The running time mainly includes the road travel time and the client-point service time as well as the queuing time and charging time at public charging stations. Thus, the time cost of EVs can be calculated using the following equation:

$$
\begin{aligned}
C_{2}= & P_{2} \cdot\left(\sum_{k \in \mathbf{K}} \sum_{i \in \mathbf{V}} \sum_{j \in \mathbf{V}} x_{i j k} t_{i j k}+\sum_{k \in \mathbf{K}} \sum_{i \in \mathbf{N}} y_{i k} t_{i}^{S}\right. \\
& \left.+\sum_{k \in \mathbf{K}} \sum_{i \in \mathbf{F}} z_{i k}\left(t_{i k}^{C}+t_{i k}^{Q}\right)\right) .
\end{aligned}
$$

(3). Energy Consumption Cost. Energy consumption cost refers to the energy consumption cost of the vehicle in the whole process of driving. Based on the calculation of power consumption above, the total energy consumption cost (C3) is

$$
C_{3}=P_{3} \cdot \sum_{k \in \mathbf{K}} \sum_{i \in \mathbf{V}} \sum_{j \in \mathbf{V}} x_{i j k} t_{i j k} E_{i j k} .
$$

(4). Charging Cost. The charging cost $\left(C_{4}\right)$ is the total cost of fast charging at public charging stations during the distribution process. Thus, the fast charging cost of EVs is

$$
C_{4}=P_{4} \cdot \sum_{i \in \mathbf{F}} \sum_{k \in \mathbf{K}}\left(E \max -E_{i k}^{A}\right) z_{i k}
$$

(5). Refrigeration Cost. The refrigeration cost of a refrigerated vehicle $\left(C_{5}\right)$ is generally the cost of refrigerating agents and energy consumption to maintain the temperature within the vehicle. According to Fang et al. [34], the refrigeration cost consists of the refrigeration cost during the transportation process and the refrigeration cost during the loading and unloading of goods: 
TABLE 3: Parameters and descriptions about correlation analysis of time-varying nature.

\begin{tabular}{lc}
\hline Parameters & Descriptions \\
\hline$T_{B}$ & The time period $B, B=\{1,2, \ldots, P\}$ \\
$t_{B}$ & The start time of time period $B, T_{B}=\left[t_{B}, t_{B+1}\right]$ \\
$v_{i j}^{B}$ & The speed that the $k^{\text {th }} \mathrm{EV}$ travels on the road $(i, j)$ at time period $\mathrm{B}(\mathrm{km} / \mathrm{h}),(i, j) \in \mathbf{R}$ \\
$D$ & The intermediate variable of the distance \\
$t_{i k}^{L}$ & The time at which the $k^{\text {th }}$ EV leaves point $i(\min ), k \in \mathbf{K}, i \in \mathbf{V}$ \\
$t l_{k}$ & The intermediate variable of travel time
\end{tabular}

$$
C_{5}=\sum_{k \in \mathbf{K}} \sum_{j \in \mathbf{V}} \sum_{i \in \mathbf{V}} P_{5} \cdot t_{i j k} x_{i j k}+\sum_{k \in \mathbf{K}} \sum_{i \in \mathbf{N}} P_{6} \cdot t_{i}^{S} \cdot y_{i k} \cdot
$$

(6). Penalty Cost. This cost $\left(C_{6}\right)$ is incurred by the vehicle as a result of violating the client's time window during the distribution process. Client $i$ accepts service outside the time window, but there is a penalty cost for failing to provide service within the appointed time, which is equal to

$$
C_{6}=p_{8} \sum_{i \in \mathbf{N}} \sum_{k \in \mathbf{K}} \max \left\{e_{i}-t_{i k}^{A}, 0\right\}+p_{9} \sum_{i \in \mathbf{N}} \sum_{k \in \mathbf{K}} \max \left\{t_{i k}^{L}-l_{i}, 0\right\} .
$$

(7). Battery Loss Cost. Fast charging will significantly reduce the service life of batteries. When the number of times a battery has fast charged exceeds the maximum number of times that it can withstand fast charging, the battery will be discarded. Thus, in this study, the battery loss cost $\left(C_{7}\right)$ is represented by a function positively correlated with the number of fast charges an EV has undergone:

$$
C_{7}=\frac{\mathrm{CE}}{\mathrm{CN} \max } \sum_{i \in \mathbf{F}} \sum_{k \in \mathbf{K}} z_{i k} \text {. }
$$

(8). Fresh Value Loss Cost. There are two primary causes of damaged goods, namely, the passage of time during the distribution and transportation process and changes in the surrounding environment during the loading/unloading and service processes. According to Fang et al. [34] and $\mathrm{Li}$ and Fan [39], a fresh-product freshness decay function is introduced to calculate the cost of damaged goods $\left(C_{8}\right)$ :

$$
\begin{aligned}
C_{81} & =\sum_{k \in \mathbf{K}} \sum_{i \in \mathbf{N}} y_{i k} \cdot P_{7} \cdot q_{i}\left(1-e^{-\partial_{1}\left(t_{i k}^{A}-t_{0 k}^{L}\right)}\right), \\
C_{82} & =\sum_{k \in \mathbf{K}} \sum_{i \in \mathbf{N}} y_{i k} \cdot P_{7} \cdot Q_{i k}^{L}\left(1-e^{-\partial_{2} t_{i}^{S}}\right), \\
C_{8} & =C_{81}+C_{82} .
\end{aligned}
$$

Equation (12) shows the fresh value loss cost during the transportation process. Equation (13) shows the fresh value loss cost when loading goods onto the vehicle and unloading goods from the vehicle.

2.3.4. Modeling. Based on the above description, a distribution route optimization model for EVs in cold chain logistics for fresh products in a time-varying road network is established, as shown below, by considering the difference in en route charging time and queuing time for charging and with the minimization of the total cost of logistic distribution that includes economic cost (consisting of the fixed EV cost, time cost, energy consumption cost, refrigeration cost, charging cost, time penalty cost, and battery loss cost) and fresh value loss cost as the objective function $Z$ :

$$
\min Z=C_{1}+C_{2}+C_{3}+C_{4}+C_{5}+C_{6}+C_{7}+C_{8},
$$

s.t.

$$
\begin{aligned}
& \sum_{k \in \mathbf{K}} \sum_{i \in \mathbf{N}} x_{i p k}=\sum_{k \in \mathbf{K}} \sum_{j \in \mathbf{N}} x_{p j k}=1, \quad \forall k \in \mathbf{K}, p \in \mathbf{N} \\
& \sum_{j \in \mathbf{N} \cup \mathbf{M}} x_{0 j k}=\sum_{i \in \mathbf{N} \cup \mathbf{M}} x_{i 0 k}=1, \quad \forall k \in \mathbf{K} \\
& \sum_{i \in \mathbf{V}} q_{j} y_{i k} \leq Q, \quad \forall k \in \mathbf{K}, \\
& E_{0 k}^{L}=E \max , \quad \forall k \in \mathbf{K}, \\
& E_{i k}^{A}\left(1-z_{i k}\right)+z_{i k} E \max =E_{i k}^{L}, \quad \forall i \in \mathbf{V}, k \in \mathbf{K} \\
& E \max \geq E_{i k}^{A} \geq E \operatorname{low}, \quad \forall i \in \mathbf{V}, k \in \mathbf{K}, \\
& E_{j k}^{A} \leq E_{i k}^{L}-E_{i j k}+M_{0}\left(1-x_{i j k}\right), \quad \forall i, j \in \mathbf{V}, k \in \mathbf{K} \\
& t_{i k}^{A}+\left(1-z_{i k}\right) * t_{i}^{S}+z_{i k}\left(t_{i k}^{Q}+t_{i k}^{C}\right)=t_{i k}^{L}, \quad \forall i \in \mathbf{V}, k \in \mathbf{K}, \\
& y_{i k} \in\{0,1\}, \quad \forall i \in \mathbf{N}, k \in \mathbf{K}, \\
& z_{i k}^{A} \in\{0,1\}, \quad \forall i \in \mathbf{F}, k \in \mathbf{K} . \\
& x_{i j k} \in\{0,1\}, \quad \forall i, j \in \mathbf{V}, k \in \mathbf{K}, \\
& t_{i j k}+M_{0}\left(1-x_{i j k}\right) \geq t_{i k}^{L}, \quad \forall i, j \in \mathbf{V}, k \in \mathbf{K}
\end{aligned}
$$

Constraint (16) indicates that each customer point can only be serviced by one EV and all customer points are serviced; constraint (17) indicates that each vehicle's starting point and returning point are the distribution center; constraint (18) indicates that the delivery vehicle's cargo weight cannot exceed the vehicle weight; constraint (19) indicates that the vehicle is fully charged when departing from the distribution center; constraint (20) indicates that the 
vehicle is fully charged when departing from the public charging station; constraint (21) indicates the vehicle's power level limits when arriving at any node, which cannot be lower than the minimal power level for normal driving or higher than the battery capacity constraint; constraint (22) indicates the power levels when the vehicle leaves node $i$ and arrives at node $j$, and M0 is a very large positive constant number; constraints (23) to (24) indicate the time constraints: constraint (23) indicates the time constraints on a vehicle arriving at and departing from node $i$, constraint (24) indicates the time relationship between the electric vehicle leaving node $i$ and arriving node $j$, and M0 is a very large positive constant number; constraints (25) to (27) indicate variable value constraints.

\section{Improved Ant Colony Algorithm (IACA) Design}

Because the ant colony algorithm has a positive feedback mechanism, strong solving ability and robustness, and can be integrated with other optimization algorithms, in this study, we modified the basic ant colony algorithm to propose an improved ant colony algorithm for solving the above problem. The corresponding parameters and descriptions of IACA are shown in Table 4.

3.1. State Transition Strategy. The ants determine the subsequent service client point $j$ through the state transition strategy based on client point $i$. The following goes over the procedure in detail.

First, the transition probability $P_{i j}^{m}$ for ant $m$ to travel from the node $i$ to the node $j$ is calculated:

$$
P_{i j}^{m}= \begin{cases}\frac{\left[\tau_{i j}\right]^{\alpha}\left[\eta_{i j}\right]^{\beta}\left[\delta_{j}\right]^{\theta}+1}{\sum_{s \in \mathbf{C}_{\mathbf{m}}^{\mathrm{T}}}\left(\left[\tau_{i s}\right]^{\alpha}\left[\eta_{i s}\right]^{\beta}\left[\delta_{s}\right]^{\theta}+1\right)}, & j \in \mathbf{C}_{\mathbf{m}}^{\mathbf{T}}, \\ 0, & \text { otherwise, }\end{cases}
$$

where $\alpha, \beta$, and $\theta$ are the weights of the pheromone concentration, the visibility, and the time-window span, respectively. According to the method proposed by Li et al. [40] for obtaining the adaptive and expected pheromone heuristic factors,

$$
\begin{aligned}
& \alpha=1+3\left(\frac{\mathrm{NC}}{\mathrm{NC} \text { max }}\right), \\
& \beta=3-2\left(\frac{\mathrm{NC}}{\mathrm{NC} \text { max }}\right) .
\end{aligned}
$$

Then, to prevent the algorithm from falling into a local optimum and avoid premature stagnation, the state transition strategy is improved based on the Roulette wheel method using the following equation:

$$
j= \begin{cases}\underset{j \in \mathbf{C}_{\mathbf{m}}^{\mathrm{T}}}{\arg \max }\left\{P_{i j}^{m}\right\}, & \forall R_{1} \leq R_{0}, \\ \text { randomly selected point } j, & \left(j \in \mathbf{C}_{\mathbf{m}}^{\mathbf{T}}\right), \\ \text { based on Roulette wheel method, } & \forall R_{1}>R_{0} .\end{cases}
$$

An algorithm parameter $R_{0}$ in the interval of $[0,1]$ is introduced. When ant $m$ selects the subsequent client point, a random number in the interval $[0,1]$ will be generated. The next client point to which the ant transitions is determined by comparing $R_{0}$ and $R_{1}$. When $R_{1} \leq R_{0}$, the point with the highest transition probability is selected as the next service client point; otherwise, the ant selects the next client point according to the procedure of the Roulette wheel method [34].

3.2. Pheromone Update Strategy. To allow the search process to be more instructive, the pheromone of the road sections composed of the routes established by all the ants upon completion of traversing is updated. The update rules are as follows:

$$
\begin{aligned}
& \tau_{i j}^{\text {new }}=\tau_{i j}^{\text {old }}(1-\sigma)+\sum_{m=1}^{M} \Delta \tau_{i j}^{m}, \\
& \Delta \tau_{i j}^{m}= \begin{cases}\frac{W}{f_{m}^{\mathrm{NC}}}, & \text { ant } m \text { travels on the } \operatorname{road}(i, j), \\
0, & \text { otherwise, }\end{cases}
\end{aligned}
$$

where $\tau_{i j}^{\text {old }}$ is the pheromone of the road section $(i, j)$ before the current iteration and $\tau_{i j}^{\text {new }}$ is the updated pheromone after the current iteration.

3.3. Charging Station Placement Strategy Based on the Minimum Power Consumption Principle. During the distribution process, an EV needs to satisfy not only the vehicle load constraint but also the power constraint. That is, the en route charging behavior of an EV needs to be taken into consideration. Therefore, when constructing a feasible solution for a single ant, it is necessary to first construct a feasible solution that satisfies the vehicle load constraint and then determine the power constraint for the EV at each client point. If the solution does not satisfy the vehicle load constraint, a charging station is placed based on the minimum power consumption principle. The following shows the procedure in detail:

(1) First, an initial feasible solution that satisfies the vehicle load constraint is generated. Then, the remaining power $E_{i k}^{A}$ is calculated when the ants reach each client point. If $E_{i k}^{A}>E$ low at all the client points, then the EV does not need to be charged en route. The operation is terminated. Otherwise, the first client point $i$ is determined where the power constraint is not satisfied and go to operation (2).

(2) Finding the charging station that requires the lowest power consumption to reach from the client point preceding client point $i$ and determining whether the remaining power after reaching this charging station satisfies the power constraint. If the constraint is satisfied, the charging station is put between the client point preceding client point $i$ and client point $i$. The EV has a remaining power of Emax after 
TABle 4: Parameters and descriptions of IACA.

\begin{tabular}{lc}
\hline Parameters & Descriptions \\
\hline$\tau_{i j}$ & The pheromone concentration, $\tau$ is the pheromone matrix \\
$\eta_{i j}$ & The visibility $\left(\eta_{i j}=\left(1 / d_{i j}\right)\right)$ \\
$\delta_{j}$ & The time-window span function $\left(\delta_{j}=\left(1 /\left[l_{j}-e_{j}\right]\right)\right)$ \\
$M$ & The total number of ants \\
$M$ & The current ant number \\
$\mathbf{C}_{\mathbf{m}}^{\mathbf{T}}$ & The maximum iteration number \\
$N C_{\max }$ & The maximum iteration number \\
$N C$ & The current iteration number \\
$\sigma$ & The volatilization factor \\
$W$ & The total pheromone strength \\
$B C$ & The global optimal value \\
$\mathbf{B R}$ & The globally optimal route \\
$f_{m}^{N C}$ & The fitness value of ant $m$ in the $N C$ iteration \\
$f_{B}^{N C}$ & The optimal fitness value in $N C$ iteration \\
$\mathrm{R}_{m}^{T}$ & The temporary set of nodes that stores the ant $m$ route \\
$E_{m}^{A}(j)$ & Temporary variable that represents the remaining electric quantity when ant $m$ reaches customer point $j$ \\
$L_{m}^{T}$ & Temporary variable that represents the current load of ant $m$ \\
$F_{m}^{A}(j)$ & Temporary variable that represents the fresh value loss of customer point $j$ when ant $m$ reaches customer point $j$ \\
$\varepsilon$ & The minimum threshold for the ratio of the fresh value loss to the fresh value \\
$V N_{m}$ & The vehicle number identification of ant $m$ \\
\hline
\end{tabular}

reaching the charging station. The $\mathrm{EV}$ goes on to fulfill the service at client point $i$. If the constraint is unsatisfied, another client point is found by forward recursion and operation (2) is repeated until finding the appropriate charging station, which is then placed.

(3) Updating the remaining power when reaching client point $i$ and all the client points following client point $i$, and go to operation (1).

The corresponding process is shown in Figure 1.

3.4. Algorithm Process. By the integration of all the steps, described above, we propose an improved ant colony algorithm for solving the distribution route optimization model for EVs in urban cold chain logistics for fresh products under time-varying traffic conditions. The optimization objective of the model is the total distribution cost. The general structure of the improved ant colony algorithm is shown in Algorithm 2, the corresponding process is shown in Figure 2, and the relevant parameters are shown in Table 4.

In order to further analyze the relationship between cost elements in the model, we take economic cost and fresh value loss cost as two targets, respectively, to solve the problem. The biobject optimization question is dealt with by the main object method [41]. The economic cost is chosen as the main objective, and the fresh value loss is taken as the constraint condition to solve the biobject optimization problem. The selection of the main objective function depends on the strategic planning of fresh e-commerce enterprises. The specific transformation is as follows:

$$
\begin{aligned}
& \min Z_{1}=C_{1}+C_{2}+C_{3}+C_{4}+C_{5}+C_{7}, \\
& \min Z_{2}=C_{8} \longrightarrow \frac{F_{m}^{A}(j)}{P_{7} \cdot d_{j}} \leq \varepsilon .
\end{aligned}
$$

The objective function (32) minimizes the economic cost; the objective function minimizing the fresh value loss cost is converted to constraint (33) by the transformation. Constraint (33) which indicates the ratio of the fresh value loss to the total fresh value of each customer point should be lower than the minimum threshold $\varepsilon$. According to the characteristics of the model, we further improve the ant colony algorithm. For each ant route selection, we added the fresh value loss ratio constraint. The specific process is shown in Algorithm 3.

\section{Simulation Tests}

4.1. Test Settings. Because of the diversity of customer distribution in fresh cold chain distribution, the data of the test example were from the international standard EV route databases [42]. Considering various factors of the EV distribution, we used the data of the type $C$ (concentrated distribution), type $\mathrm{R}$ (random distribution), and type $\mathrm{RC}$ (mixed distribution) examples of the international standard of the EVRPTW database. In each example, there are 20 charging stations (No.1 to No.20), 100 customer points (No.21 to No.120), and 1 distribution center (No.0). Considering that there are multiple scenarios for the coordinates of fresh-product cold chain distribution clients, (1) the starting and ending times of the model are set to 7:00 (corresponding to time 0, i.e., $0 \mathrm{~min}$ ) and 24:00 (corresponding to 1,020 min), respectively. The length of each time period is $10 \mathrm{~min}$. According to urban traffic patterns, the period 7:30-9:00 (i.e., $4^{\text {th }}-12^{\text {th }}$ periods) and the period 17: 30-19:00 (i.e., $64^{\text {th }}-72^{\text {nd }}$ periods) are set as traffic congestion periods (vehicle speeds vary with the road-section type), and the other periods are set as normal periods. According to Xiao and Konak [37], let $\omega=0.1$. Three vehicle speeds $[\operatorname{VR}(1-\omega), \operatorname{VR}(1+2 \omega), \operatorname{VR}(1-3 \omega)]$ are allowed. Based on the $T$ value of the time period, the remainder function 


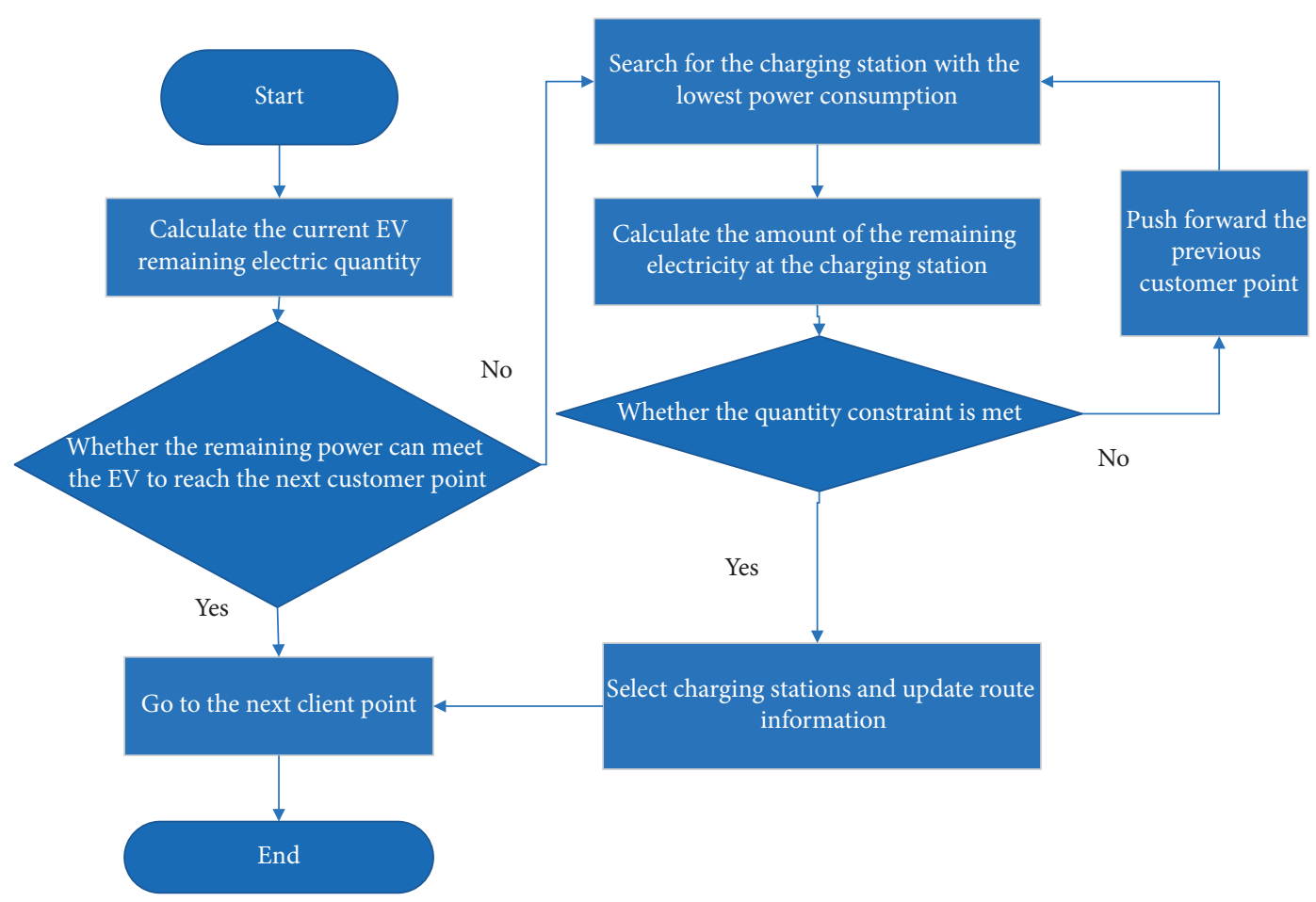

FIGURE 1: Charging strategy flowchart.

(i) Input: traffic network information, electric vehicle information, customer points, charging station related information, etc.

(ii) Output: optimal path $\mathrm{BR}$ and optimal value $\mathrm{BC}$

(1) Set $M, N C_{\max }, \alpha, \beta, \theta$, etc.

(2) $N C=1$

(3) while $\mathrm{NC} \neq \mathrm{NC}_{\max }$

(4) Initialize the ant colony path $R_{m}^{T}$, pheromone matrix $\tau \mathbf{C}_{\mathbf{m}}^{\mathbf{T}}$. All ants go back to the distribution center

(5) For $m=1: \mathrm{M}$

(6) while $C_{m}^{T} \neq \varnothing$

(7) Select customer point $j$ according to state transition strategy

(8) $\quad$ if $L_{m}^{T}+q_{j} \leq \mathrm{Q}$

(9) $\quad$ if $E_{m}^{A}(j)<E_{\text {low }}$

(10) Insert the charging station in the route according to the minimum power consumption principle and update the $R_{m}^{T}$ information

(11) end if

(12) Insert the customer point $j$ to the end of the route $R_{m}^{T}$, update tabu list, delete $j$ from $\mathbf{C}_{\mathrm{m}}^{\mathbf{T}}$. If $j$ is the first service node, calculate the departure time.

(13) $\quad$ else $L_{m}^{T}+q_{j}>Q$

(14) Ant $m$ returns to the distribution center, insert 0 to the end of $R_{m}^{T}, \mathrm{VN}_{m}=\mathrm{VN}_{m}+1, L_{m}^{T}=0$

(15) end if

(16) end while

(17) end for

(18) Calculate the fitness of each ant and select the optimal value for this iteration $f_{B}^{\mathrm{NC}}$

(19) Update the segment pheromones according to each ant's path and fitness

(20) if $f_{B}^{\mathrm{NC}}<\mathrm{BC}$

(21) $\quad \mathrm{BC}=f_{B}^{\mathrm{NC}}$, update $\mathbf{B R}$

(22) end if

(23) $\mathrm{NC}=\mathrm{NC}+1$

(24) end while

(25) return $B C, B R$

Algorithm 2: IACA for the model that targets the total cost of distribution. 


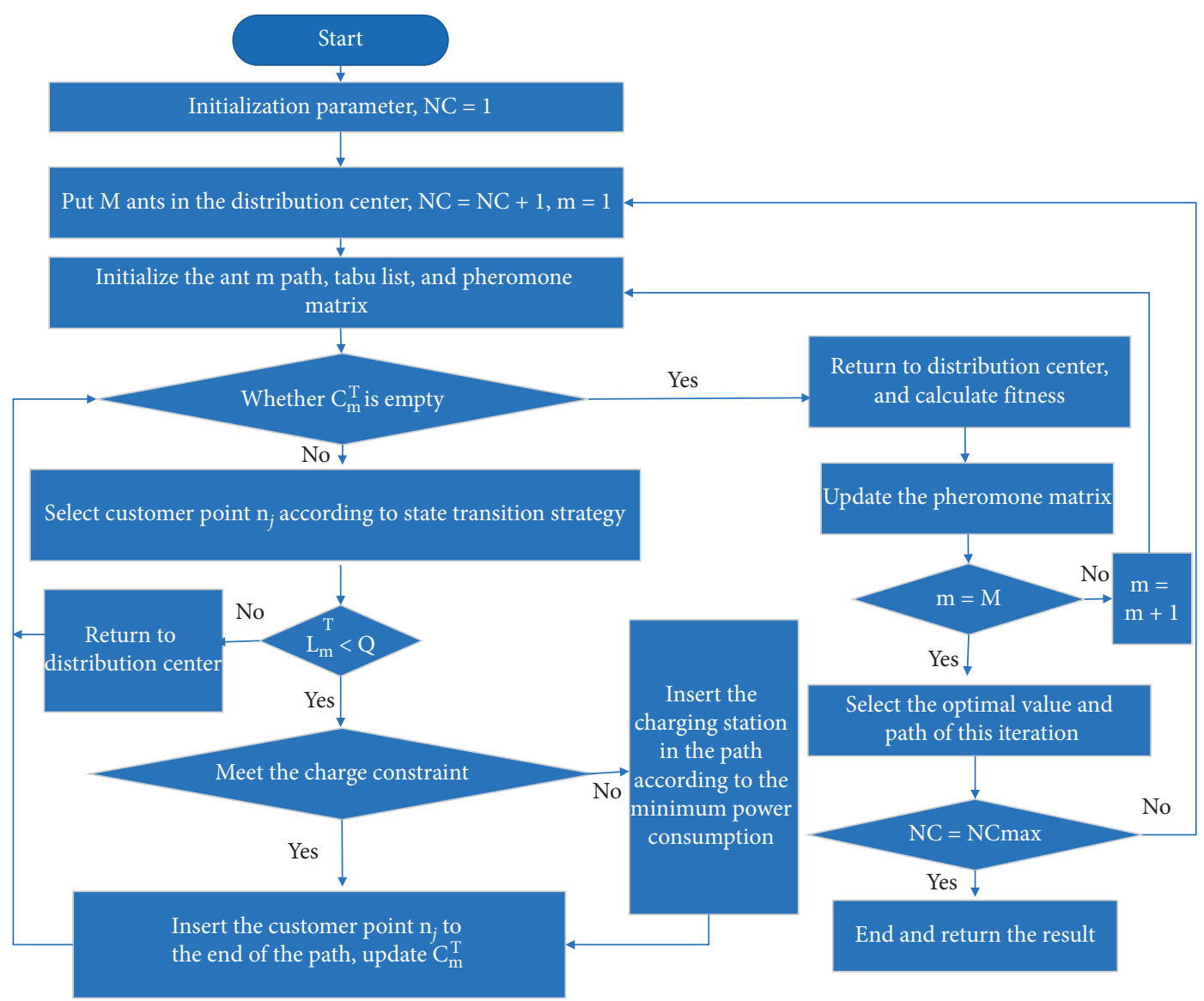

FIGURE 2: IACA for the model that targets the total cost of distribution.

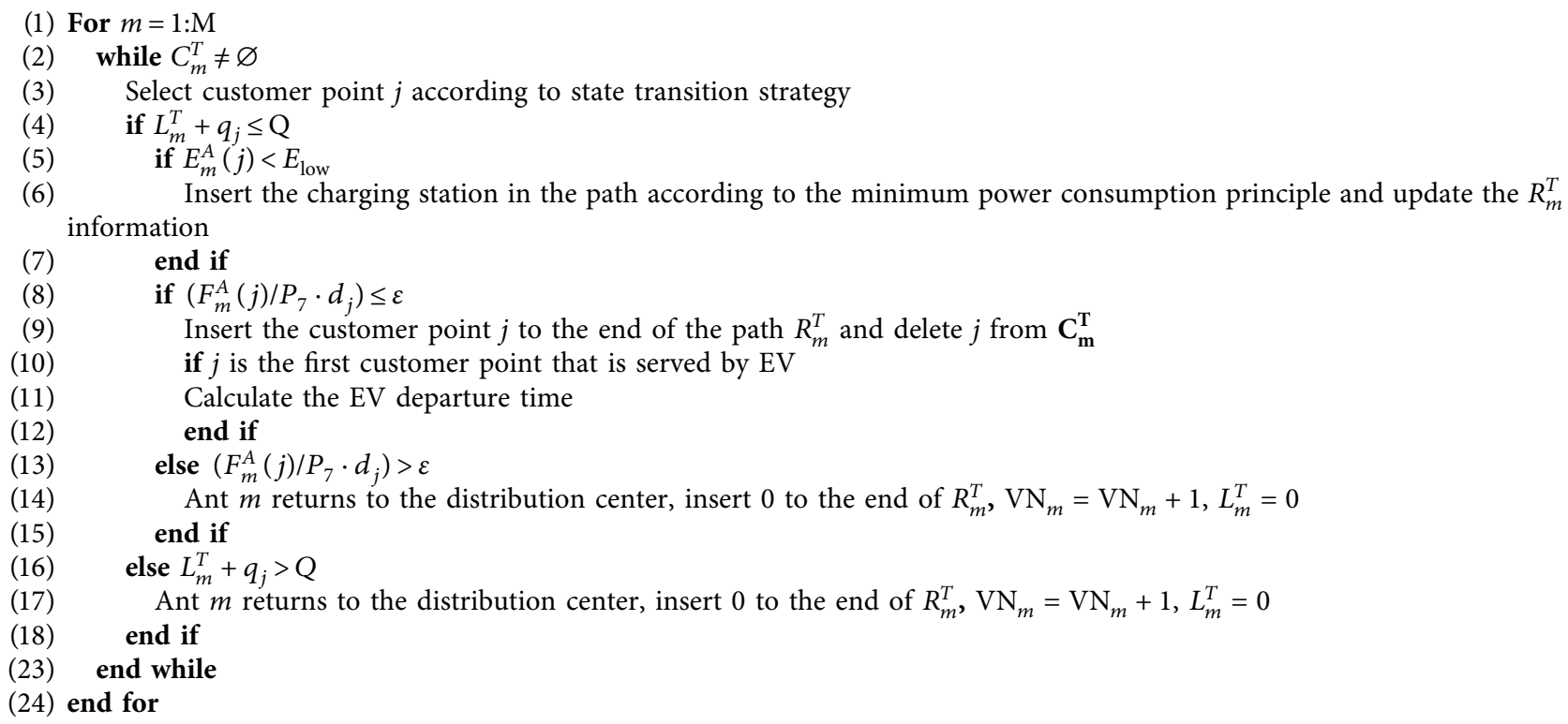

Algorithm 3: Intermediate process of IACA for the optimization model with the fresh value loss constraint. 
$\pi=\bmod (T, 3)$ is used. The $\pi$ values of 0,1 , and 2 correspond to three vehicle speeds during the normal time periods. The vehicle speeds vary with the road type. (2) In the actual distribution, the nodes are not connected by straight roads but by multiple roads; therefore, using the straight line distance instead of the distance between two points will lead to quite different from the actual situation. This paper introduces the circuitous coefficient $\delta(\delta=1.5)$ [43], and $d_{i j}$ is the product of the straight-line distance between two points and the circuitous coefficient. (3) To be more consistent with real life, the areas where any nodes are located within the road network are classified into three types with urban inner-ring highways as boundaries $((40,40)$ is the center of each inner ring; the radii of the second and third rings of the city are 20 and 30, respectively). The area within the second ring is denoted by $A_{1}$. The area between the second and third rings is denoted by $A_{2}$. The area outside the third ring is denoted by $A_{3}$. (4) Based on the area where any two arbitrary nodes are located, road sections are classified into six types. Table 5 summarizes the attributes of the road sections.

The vehicle had an unloaded weight of $4000 \mathrm{~kg}$, with a capacity of 200 weight units; assuming that one weight unit is $10 \mathrm{~kg}$, the vehicle's load capacity is 2 tons. The model parameters are shown in Table 6, and the algorithm parameters are shown in Table 7. The calculation was performed using MATLAB R2016 on a PC with a $2.0 \mathrm{GHz}$ CPU and $8 \mathrm{G}$ memory.

4.2. Analysis of the Stability of the Algorithm, Charging Planning, and Departure Time Based on a Large-Scale Dataset. To examine its stability, the proposed algorithm was tested on the R204 dataset. The program was run 10 times. The average running time was $378.491 \mathrm{~s}$. This demonstrates that the proposed algorithm can produce an optimum EV route planning scheme within a relatively short time. Figure 3 shows the optimum EV route planning based on the R204 dataset (the cyan and brown rings are the second and third rings of the city, respectively).

The R204 dataset was used in the example, and the detailed route optimization scheme is shown in Table 8. In Table 8, "VN" represents the vehicle number; "EVR" represents the vehicle route; 0 stands for the distribution center, 1-20 for the charging stations, and 21-120 for the customer points; "ST" represents the EV departure time/(min); "ET" represents the end of the EV distribution time; "CN" represents the number of times EV is fast charging; "CT" represents the EV charging time/(min); "QT" represents the EV queuing time for charging/(min); "TT" represents the total EV travel time/( $\mathrm{min})$. The test calculation results show the following. The total distribution cost is 5,579.2 yuan. Eight EVs are used. The travel distance is $1,164.6 \mathrm{~km}$. The fixed, travel, refrigeration, fresh value loss, energy consumption, charging, penalty, and battery loss costs are 1,600, $1,429.3,397.4,741.3,238.2,187.2,760.8$, and 225 yuan, respectively. Table 8 summarizes the routes and times in detail. The following can be derived from Table 8. (1) A comprehensive analysis of the EVR and the $\mathrm{CN}$ finds that there is a significant difference in the distribution route between EVs due to limiting factors (e.g., time-varying road network conditions, load, and battery capacity). The $5^{\text {th }}$ and $7^{\text {th }}$ EVs have the most distribution tasks, serving 15 clients. The $8^{\text {th }}$ EV has the fewest, only 9 clients. The $5^{\text {th }}, 6^{\text {th }}, 7^{\text {th }}$, and $8^{\text {th }}$ EVs need to be charged at public charging stations during the distribution process so that they can continue to complete their distribution tasks, whereas the other EVs do not need to be charged during the distribution process. This suggests that to complete their distribution tasks, EVs need to go to public charging stations for charging to increase their single-charge mileage and expand their distribution area within a short time. (2) An analysis of the ST finds that the $1^{\text {st }}, 2^{\text {nd }}, 3^{\text {rd }}, 4^{\text {th }}, 6^{\text {th }}, 7^{\text {th }}$, and $8^{\text {th }}$ EVs depart at time 0 from the distribution center to distribute goods, while the $5^{\text {th }} \mathrm{EV}$ departs at the other time. If departing at time 0 , an EV needs to bear a relatively high time-window penalty cost. This suggests that a logistic enterprise needs to scientifically plan routes based on the actual conditions (e.g., road network conditions and time windows at client points). (3) An analysis of the QT and CT finds that the $5^{\text {th }}, 6^{\text {th }} 7^{\text {th }}$, and $8^{\text {th }}$ EVs extend their passage range by fast charging, but the travel time of EV is increased due to charging time and queuing time. It is obvious that in all charged EVs, the highest proportion of queuing time to the travel time is $6.6 \%$ $\left(5^{\text {th }} \mathrm{EV}\right)$, and the smallest proportion is $4.7 \%\left(7^{\text {th }} \mathrm{EV}\right)$. The highest proportion of charging time to the travel time is $7.9 \%$ $\left(6^{\text {th }} \mathrm{EV}\right)$, and the smallest proportion is $6.3 \%\left(7^{\text {th }} \mathrm{EV}\right)$. In addition, the highest proportion of the sum of charging time and queuing time to the travel time is $13.9 \%$. Therefore, in the distribution process, charging time and queuing time cannot be ignored. (4) An analysis of the ST and ET finds that the $1^{\text {st }}, 2^{\text {nd }}, 3^{\text {rd }}, 4^{\text {th }}, 6^{\text {th }}, 7^{\text {th }}$, and $8^{\text {th }}$ EVs operate during the morning peak congestion time period only, and the $5^{\text {th }}$ $\mathrm{EV}$ operates during the evening peak congestion time period only. This demonstrates that the proposed algorithm can relatively effectively avoid congestion time periods and improve the distribution efficiency of vehicles.

4.3. Analysis of Vehicle Path Planning for Various Types of Cases. Fresh-product electronic commerce enterprises have various types of clients distributed in various patterns. In view of this, solutions are found based on various Solomon datasets. In the type- $\mathrm{C}$ dataset, the clients are distributed in a concentrated area, the time windows are less than $1 \mathrm{~h}$, and the client service time is relatively long $(90 \mathrm{~min})$. In the type$\mathrm{R}$ dataset, the clients are distributed randomly, the time windows are greater than $1 \mathrm{~h}$, and the service time is relatively short $(10 \mathrm{~min})$. In the type-RC dataset, the clients are distributed in a mixed manner, most of the time windows are $30 \mathrm{~min}$, and the service time is $10 \mathrm{~min}$. Figure 4 shows the optimal route map based on various datasets.

The experimental results are shown in Table 9. In Table 9, "EX" is the dataset type, "TC" is the total distribution cost (yuan), "VD" is the total travel distance $(\mathrm{km})$, "ECC" is the energy consumption and charging cost (yuan), "TMC" is the time management cost (yuan) (including the time cost and the penalty cost), "RC" is the refrigeration cost (yuan), "VL" is the cost of fresh value loss (yuan), "VN" is the number of 
TABLE 5: Attributes of various road sections.

\begin{tabular}{lcc}
\hline Road-section type & Road-section describe & Vehicle speeds at different periods $(\mathrm{km} / \mathrm{h})$ \\
\hline$?$ & $i \in A_{1}, j \in \mathbf{A}_{1}$ & Normal periods: VR $=30$, three speeds: $(27,36,21) ;$ congestion periods: 20 \\
$?$ & $i \in \mathbf{A}_{1}, j \in \mathbf{A}_{2}$ & Normal periods: VR $=40$, three speeds: $(36,48,28) ;$ congestion periods: 20 \\
$?$ & $i \in \mathbf{A}_{2}, j \in \mathbf{A}_{2}$ & Normal periods: VR $=50$, three speeds: $(45,60,35) ;$ congestion periods: 30 \\
$?$ & $i \in \mathbf{A}_{1}, j \in \mathbf{A}_{3}$ & Normal periods: $\mathrm{VR}=60$, three speeds: $(54,72,42) ;$ congestion periods: 30 \\
$?$ & $i \in \mathbf{A}_{2}, j \in \mathbf{A}_{3}$ & Normal periods: VR $=70$, three speeds: $(63,84$, 49); congestion periods: 40 \\
$?$ & $i \in \mathbf{A}_{3}, j \in \mathbf{A}_{3}$ & Normal periods: VR $=80$, three speeds: $(72,96,56) ;$ congestion periods: 40 \\
\hline
\end{tabular}

TABle 6: Model parameters.

\begin{tabular}{|c|c|c|}
\hline Parameter & Description & Value \\
\hline$E_{\max }$ & The EV maximum battery capacity (kWh) & 60 \\
\hline$E_{\text {low }}$ & The EV minimum battery power level (kWh) & 12 \\
\hline$P_{1}$ & Fixed cost of per EV (yuan) & 200 \\
\hline$P_{2}$ & Travel cost of EV per unit time (yuan/min) & 0.5 \\
\hline$P_{3}$ & Cost per unit electricity consumption (yuan/kWh) & 0.5 \\
\hline$P_{4}^{3}$ & Unit electricity price of fast charging (yuan $/ \mathrm{kWh}$ ) & 1 \\
\hline$P_{5}$ & Refrigeration costs during EV transportation process of unit time (yuan/h) & 5 \\
\hline$P_{6}$ & Refrigeration costs during EV unloading process of unit time (yuan/h) & 12 \\
\hline$P_{7}$ & Price of the unit commodity (yuan $/ \mathrm{kg}$ ) & 10 \\
\hline$P_{8}$ & Cost of waiting for the unit time if refrigerated $\mathrm{EV}$ arrives at customer node in advance (yuan/min) & 0.3 \\
\hline$P_{9}$ & Cost of punishing for the unit time if refrigerated $\mathrm{EV}$ is late to the customer node (yuan/min) & 0.3 \\
\hline$s_{i}$ & The number of charging piles in the charging station $I, i \in \mathbf{F}$ & 10 \\
\hline$\lambda_{i}$ & The number of EVs that reach the charging station $i$ (vehicles/h) & $\begin{array}{c}\text { Congestion periods: } \\
25 \\
\text { Normal periods: } 15 \\
\text { Congestion periods: }\end{array}$ \\
\hline$\mu_{i}$ & The number of EVs that have completed charging per unit time in the charging station $i$ (vehicles/h) & $\begin{array}{l}3 \\
\text { Normal periods: } 2\end{array}$ \\
\hline$\omega_{A_{i}}$ & $\begin{array}{l}\text { Area factor for charging demand which is introduced to differentiate the charging demand between } \\
\text { different areas, } A_{i} \in \mathbf{A}\end{array}$ & $\begin{array}{c}A_{1}: 1.1 \\
A_{2}: 1 \\
A_{3}: 0.9\end{array}$ \\
\hline$r_{c}$ & Charging speed of the public charging station $(\mathrm{kWh} / \mathrm{min})$ & 1.5 \\
\hline$C N_{\max }$ & The maximum number of charging cycles & 800 \\
\hline$C E$ & Cost of a battery (yuan) & 45000 \\
\hline$\partial_{1}$ & Freshness decay coefficient during the transportation process & 0.01 \\
\hline$\partial_{2}$ & Freshness decay coefficient during the unloading process & 0.02 \\
\hline$g$ & The acceleration of gravity $\left(\mathrm{m} / \mathrm{s}^{2}\right)$ & 9.8 \\
\hline$\eta$ & The mechanical efficiency of the transmission system & 0.9 \\
\hline$f$ & The rolling resistance coefficient & 0.01 \\
\hline$C_{d}$ & The air resistance coefficient & 0.7 \\
\hline$W_{a}$ & The windward area of the vehicle $\left(\mathrm{m}^{2}\right)$ & 5.9 \\
\hline
\end{tabular}

TABLE 7: Ant colony algorithm parameters.

\begin{tabular}{lcc}
\hline Parameter & Description & Value \\
\hline NCmax & The maximum iteration number & 400 \\
$\alpha$ & Weight of the pheromone concentration & 1 \\
$\beta$ & Weight of the visibility & 3 \\
$\theta$ & Weight of the time-window span & 2 \\
$\mathrm{M}$ & The total number of ants & 30 \\
$\sigma$ & The volatilization factor & 0.2 \\
$\mathrm{~W}$ & The total pheromone strength & 100 \\
$R_{0}$ & Algorithm parameter in the interval of $[0,1]$ & 0.7 \\
\hline
\end{tabular}

vehicles, and "CPUT" is the running time (s). Table 9 shows the following. (1) The longest running time is only $395.1 \mathrm{~s}$. This suggests that the proposed algorithm can produce an effective optimization result for each dataset within a short time. (2) The TC, TMC, RC, VL, and VN for the type-C dataset are the highest. This is primarily because the service time for all the type-C clients is $90 \mathrm{~min}$ (compared to the service time of $10 \mathrm{~min}$ for the R- and RC-type clients), and the total distribution time is relatively long. Consequently, the $\mathrm{TMC}, \mathrm{VL}$, and $\mathrm{RC}$, which are related to the total 


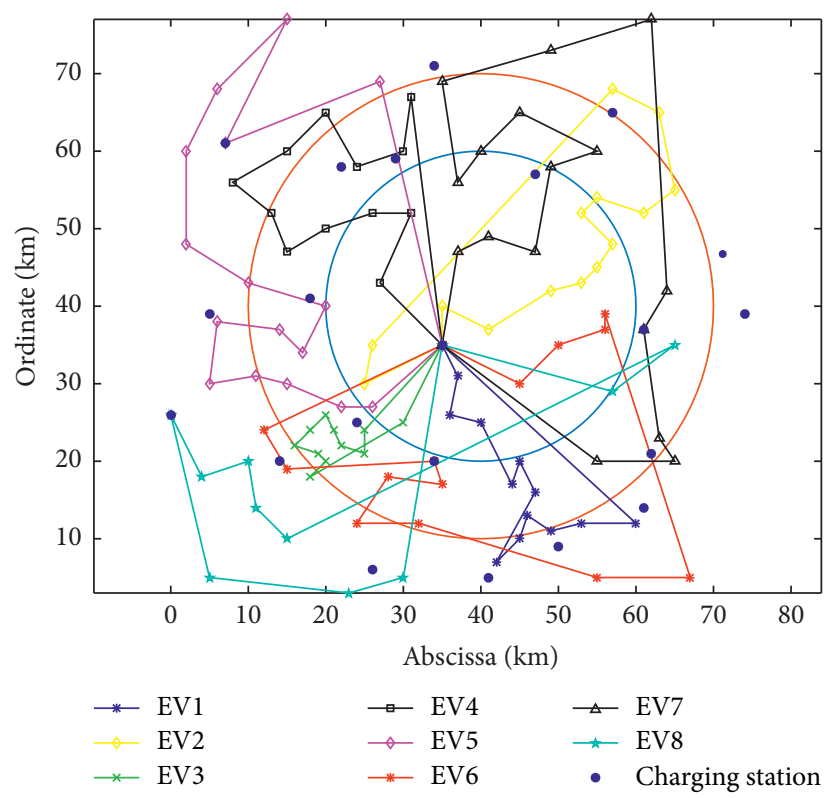

FIgURE 3: Vehicle route planning of the example R204.

TABLE 8: Vehicle route optimization results of the example with the R204 dataset.

\begin{tabular}{lccccrrr}
\hline VN & EVR & ST & ET & CN & CT(CT/TT) & QT(QT/TT) & TT \\
\hline 1 & $0-73-78-60-93-41-92-61-42-94-95-76-59-0$ & 0 & 257.70 & 0 & $0(0)$ & $0(0)$ & 257.70 \\
2 & $0-47-48-96-97-23-99-53-101-98-54-55-91-109-26-0$ & 0 & 356.34 & 0 & $0(0)$ & $0(0)$ & 356.34 \\
3 & $0-33-120-57-118-105-113-119-79-112-117-115-0$ & 0 & 220.19 & 0 & $0(0)$ & $0(0)$ & 220.19 \\
4 & $0-72-51-108-27-102-68-67-39-31-82-30-110-0$ & 0 & 317.56 & 0 & $0(0)$ & $0(0)$ & 317.56 \\
5 & $0-114-116-25-104-37-65-103-80-38-28-66-56-69-84-8-83-0$ & 509.60 & 946.37 & 1 & $32.01(7.3 \%)$ & $29.02(6.6 \%)$ & 436.77 \\
6 & $0-46-32-100-88-87-43-77-62-107-22-15-111-81-0$ & 0 & 399.42 & 1 & $31.54(7.9 \%)$ & $24.26(6.0 \%)$ & 399.42 \\
7 & $0-89-21-70-71-29-40-50-90-52-86-85-49-1-75-45-24-0$ & 0 & 498.56 & 1 & $31.32(6.3 \%)$ & $23.65(4.7 \%)$ & 498.56 \\
8 & $0-74-44-34-64-36-106-12-58-63-35-0$ & 0 & 372.17 & 1 & $29.26(7.8 \%)$ & $19.58(5.2 \%)$ & 372.17 \\
\hline
\end{tabular}

"VN" represents the vehicle number; "EVR" represents the vehicle route; 0 stands for the distribution center, 1-20 for the charging stations, and 21-120 for the customer points; "ST" represents the EV departure time; "ET" represents the end of the EV distribution time; "CN" represents the number of times EV is fast charging; "CT" represents the EV charging time; "QT" represents the EV queuing time for charging; "TT" represents the total EV travel time.

distribution time, are relatively high, resulting in the highest TC. (3) The VD and ECC for the type-C dataset are lower than those for the type- $\mathrm{R}$ and - $\mathrm{RC}$ datasets. This is mainly because in the type- $C$ dataset, the clients are distributed in a relatively concentrated area, so the travel distance and time are relatively short during the distribution process, resulting in relatively low power consumption. (4) In these datasets, the ECC accounts for a very small proportion of the TC, with the highest proportion being 7.8\% (in the R203-type dataset). In comparison, the TMC, RC, and VL, which are related to the delivery time, each account for a relatively large proportion of the TC. The highest proportion of the sum of these three costs to the TC is $80.0 \%$ (in the C103-type dataset), and the smallest is $60.0 \%$ (in the R204-type dataset). This suggests that reducing transportation time is the key to effectively reducing the cold chain transportation cost of EVs. Therefore, how to improve the single-charge mileage of EVs and reduce their charging time and frequency is the key to reducing the distribution cost of EVs in cold chain logistics for fresh products.
4.4. Analysis of Vehicle Route Planning for Various Optimization Objectives. The route planning produced by the proposed algorithm is compared with that produced with the minimization of the total travel distance of vehicles as an objective. Table 10 summarizes the test results. In Table 10, "min TC" is the minimization of the total distribution cost (yuan), "min VD" is the minimization of the total travel distance $(\mathrm{km})$, and "min PC" is the minimization of energy consumption (kWh). The meanings of "EX," "TC," "VD," and "ECC" are the same as in Table 9.

Table 10 shows the following. (1) The proposed model can produce a lower total cost than models with other objectives. (2) In the type-C dataset, the TC values obtained with the three objectives differ relatively insignificantly. The TC obtained with the minimization of the VD as the objective is $4.2 \%$ higher than that produced by the proposed algorithm. The TC obtained with the minimization of the PC as the objective is only $3.4 \%$ higher than that produced by the proposed algorithm. In comparison, in the type- $\mathrm{R}$ and type$\mathrm{RC}$ datasets, the TC values obtained with the objectives differ 

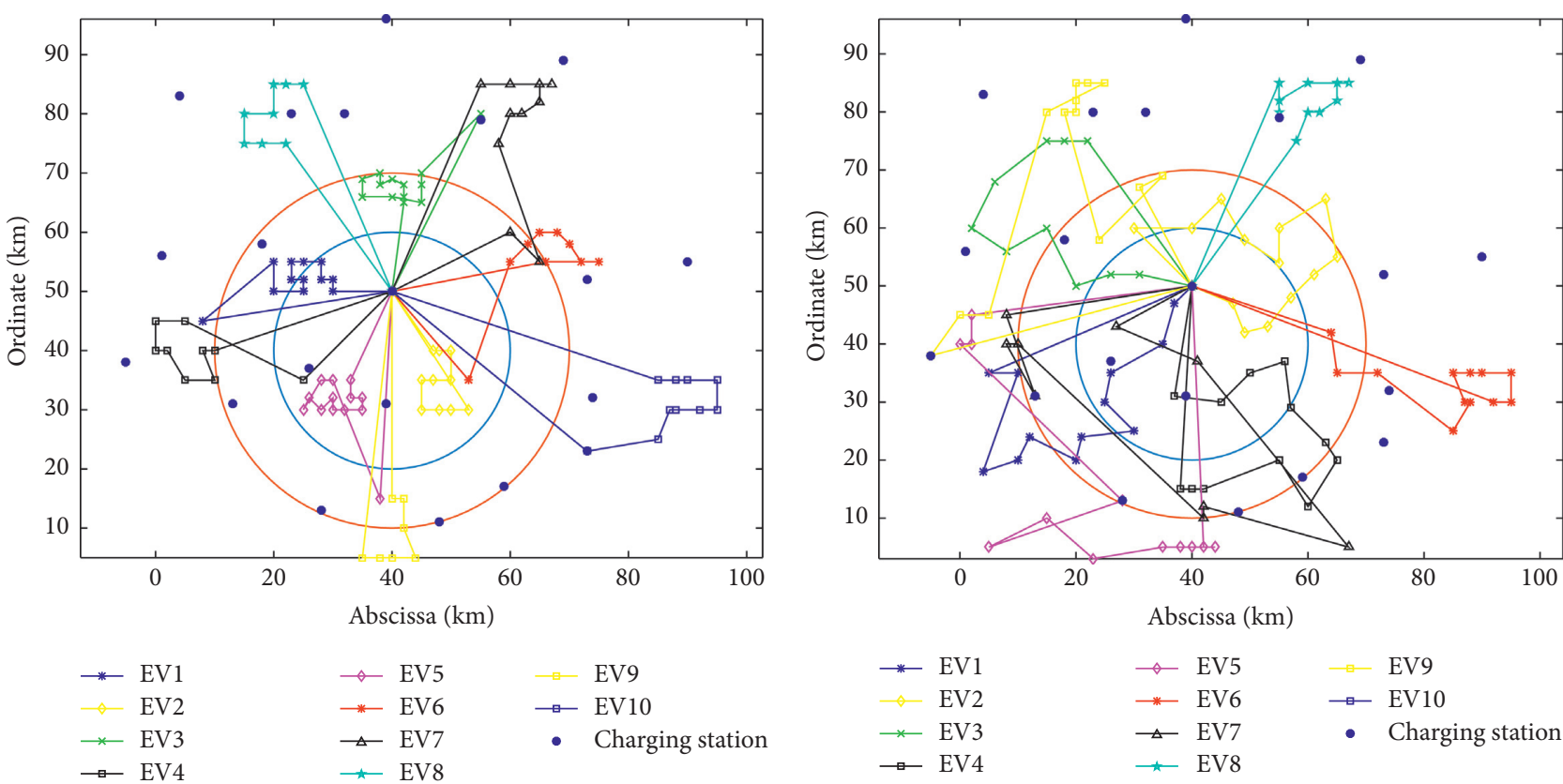

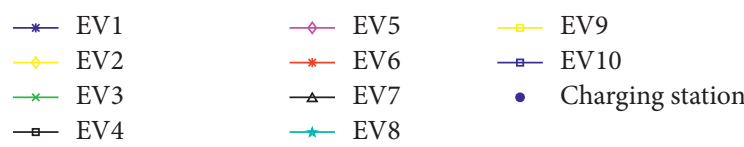

(a)

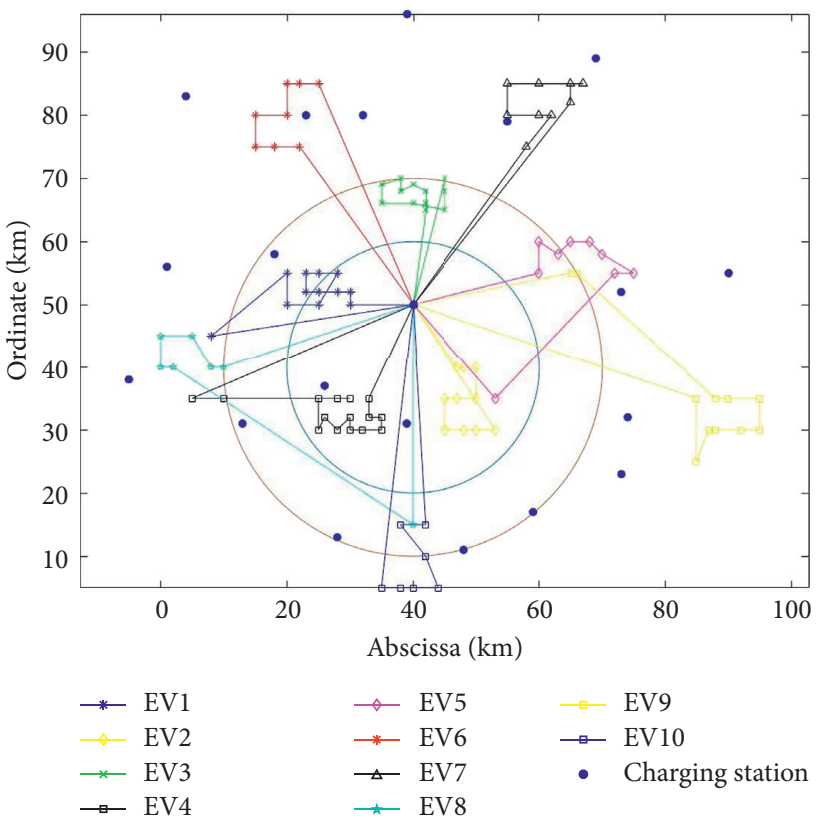

(c)

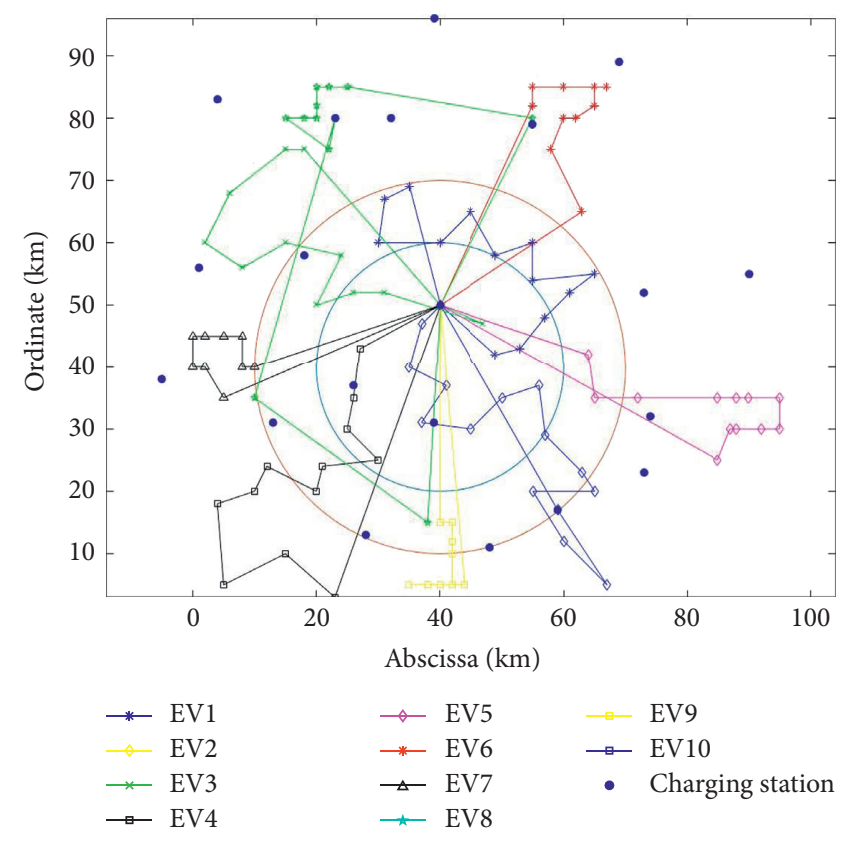

(d)

Figure 4: Route planning based on various datasets. (a) Route planning based on C103. (b) Route planning based on RC203. (c) Route planning based on C104. (d) Route planning based on RC204.

TABLE 9: Simulation results of examples with different customer distributions.

\begin{tabular}{lcccccccc}
\hline EX & TC & VD & ECC & TMC & RC & VL & VN & CPUT \\
\hline C103 & $11,805.7$ & 998.2 & 311.2 & $5,118.2$ & $1,696.7$ & $2,623.30$ & 10 & 395.1 \\
C104 & $11,745.1$ & $1,043.1$ & 295.7 & $5,091.6$ & $1,702.8$ & $2,598.70$ & 10 & 393.7 \\
R203 & $5,873.80$ & $1,287.8$ & 461.2 & $2,405.3$ & 410.7 & 771.6 & 8 \\
R204 & $5,579.40$ & $1,164.6$ & 425.3 & $2,190.4$ & 397.4 & 741.3 & 8 & 381.2 \\
RC203 & $6,865.8$ & $1,317.8$ & 431.2 & $3,123.1$ & 527.2 & 871.8 & 9 & 378.5 \\
RC204 & $6,712.4$ & $1,240.2$ & 443.1 & $3,042.3$ & 501.8 & 812.7 & 9 \\
\hline
\end{tabular}

"EX" is the dataset type, "TC" is the total distribution cost (yuan), "VD" is the total travel distance (km), "ECC" is the energy consumption and charging cost (yuan), "TMC" is the time management cost (including the travel cost and the penalty cost) (yuan), "RC" is the refrigeration cost (yuan), "VL" is the cost of fresh value loss (yuan), "VN" is the number of vehicles, and "CPUT" is the running time (s). 
TABLE 10: Calculation results based on various datasets with various optimization objectives.

\begin{tabular}{|c|c|c|c|c|c|c|c|c|c|}
\hline \multirow{2}{*}{ EX } & \multicolumn{3}{|c|}{$\min \mathrm{TC}$} & \multicolumn{3}{|c|}{$\min \mathrm{VD}$} & \multicolumn{3}{|c|}{$\min P C$} \\
\hline & TC & VD & ECC & TC & VD & ECC & TC & VD & ECC \\
\hline C104 & $11,745.1$ & $1,043.1$ & 295.7 & $12,236.3$ & 959.1 & 261.0 & $12,143.9$ & 962.4 & 249.9 \\
\hline R204 & $5,579.40$ & $1,164.6$ & 425.3 & $6,512.1$ & $1,012.4$ & 401.9 & $6,634.4$ & $1,027.8$ & 389.5 \\
\hline RC204 & $6,712.4$ & $1,240.2$ & 443.1 & $7,696.2$ & $1,148.6$ & 416.4 & $7,535.8$ & $1,183.1$ & 408.5 \\
\hline
\end{tabular}

"min TC" is the minimization of the total distribution cost (yuan), "min VD" is the minimization of the total travel distance (km), and "min PC" is the energy consumption (kWh). The meanings of "EX," "TC," "VD," and "ECC" are the same as in Table 9.

relatively significantly. For example, in the R204-type dataset, the TC obtained with the minimization of the PC as the objective is $18.9 \%$ higher than that produced by the proposed algorithm, and in the RC204-type dataset, the TC obtained with the minimization of the VD as the objective is $14.6 \%$ higher than that produced by the proposed algorithm.

4.5. Analysis on the Economic Cost Analysis under the Different Constraints of Fresh Value Loss. To analyze the relationship between cost elements in the model, we take economic cost and fresh value loss cost as two targets, respectively, to solve the problem. We use the main object method to deal with it. The economic cost is the main object, and the fresh value loss cost is a secondary goal that is converted to the constraint (the ratio of fresh value loss to total value of each customer point should be lower than the minimum threshold). The experiment was tested on the R204 dataset. Table 11 summarizes the test results. In Table 11 , " $\varepsilon$ " is the minimum threshold for the ratio of the fresh loss value to the fresh value, "TC" is the total distribution cost (yuan), "EC" is the economic cost (yuan), "VL" is the cost of fresh value loss (yuan), "VN" is the number of vehicles, and "CN" represents the number of times EV is fast charging.

Table 11 shows the following. (1) As the proportion of fresh value loss decreases, the fresh value loss cost decreases, but the total cost and economic cost increase. Because of the improvement of customers' requirements on the quality of fresh goods, the travel time and the number of customers serviced of each EV are relatively reduced, so more electric vehicles are needed for distribution. (2) When the proportion of fresh value loss is reduced, the number of customers served, travel time, and the travel distance of each EV are reduced; therefore, the probability of en routing charging decreases. In addition, when the proportion is less than $5 \%$, EVs do not need en route charging.

4.6. Comparative Analysis of Various Algorithms. To examine its effectiveness, the proposed algorithm (IACA) is compared with the classical ACA and the tabu search algorithm (TSA). In the classical ACA designed by Devapriya et al. [20] and the TSA designed by Li and Zhang [44], the departure time for all the used vehicles is 0 . In the classical ACA, there is no time-window span factor or an adaptive operator in the state transition rule. The R204 and RC204 datasets are used in the test. Table 12 summarizes the results. "EX," "TC," "VD," and "CPUT" are the same as in Table 9.
TABLE 11: Calculation results obtained using two algorithms based on various datasets.

\begin{tabular}{lccccc}
\hline$\varepsilon(\%)$ & TC & EC & VL & VN & CN \\
\hline 10 & 5579.4 & 4838.1 & 741.3 & 8 & 4 \\
7 & 5621.7 & 4943.9 & 677.8 & 10 & 1 \\
5 & 5813.2 & 5182.8 & 630.4 & 12 & 0 \\
3 & 6250.4 & 5850.6 & 399.8 & 15 & 0 \\
\hline
\end{tabular}

TABLE 12: Calculation results obtained using different algorithms based on various datasets.

\begin{tabular}{lcccc}
\hline EX & Algorithm & TC & VD & CPUT \\
\hline \multirow{4}{*}{ R204 } & IACA & $5,579.4$ & $1,164.6$ & 378.5 \\
& ACA & $5,957.1$ & $1,225.4$ & 369.3 \\
& TSA & $6,022.5$ & $1,322.7$ & 351.7 \\
\multirow{2}{*}{ RC204 } & IACA & $6,712.4$ & $1,240.2$ & 388.8 \\
& ACA & $7,168.9$ & 1309.1 & 379.9 \\
& TSA & $7,298.4$ & 1386.8 & 360.2 \\
\hline
\end{tabular}

"TC" is the total distribution cost (yuan), "VD" is the total travel distance $(\mathrm{km})$, and "CPUT" is the running time (s).

The algorithms take the total distribution cost as the target to solve the EVRP. The following can be derived from Table 12. (1) The proposed algorithm (IACA) outperforms the ACA and the TSA in terms of TC and VD. (2) In regard to computational time, while the proposed algorithm takes into consideration the calculation of the departure time and the improvement of the state transition probability, which are not considered in the ACA and the TAS, there is no significant difference in computation time of the three algorithms. The IACA can produce an optimal result within a relatively short time. (3) Introducing the adaptive heuristic operator and the expected heuristic factor effectively improves the local search and global convergence performance of the ACA.

\section{Conclusion}

The characteristics of EVs used in urban cold chain distribution of fresh products are analyzed. On this basis and in view of the time-varying nature of urban road network traffic and the overall power deficiency in EVs, a distribution route optimization model for EVs in urban cold chain logistics for fresh products in time-varying urban traffic networks is established with the minimization of the total cost (including economic cost and fresh value loss cost) as an objective. This model accounts for the differences between urban areas, road types, and queuing times at charging stations. This 
model also satisfies load, power, and client time-window constraints. Moreover, a corresponding ACA is designed to solve the model. Furthermore, the state transition strategy and pheromone update are improved.

Results obtained from multiple tests show the following. (1) When using EVs in cold chain distribution, it is necessary to reasonably plan their distribution routes based on the actual conditions (e.g., urban traffic network, client's time window, power consumption and battery capacity of EVs, and public charging stations) to rationally avoid congestion time periods and reduce the distribution cost. (2) Reducing the distribution time is the key to reducing the cost of cold chain transportation of fresh products. When using EVs in urban cold chain distribution of fresh products, it is important to reduce charging time and frequency by means such as improving battery capacity and increasing singlecharge mileage, to effectively reduce cost. (3) With the increase of customers' requirement for fresh value loss cost reduction, the total cost will increase accordingly. Therefore, it is necessary to scientifically plan the distribution route according to the specific requirements of customers to improve customer satisfaction. (4) The model and improved ACA presented in this study can be used to rationally plan distribution time, effectively avoid traffic congestion periods, and reduce the distribution cost.

However, in the actual operation of urban cold chain distribution of fresh products, logistic enterprises face a more complex environment, such as the fresh commodity diversity, the uncertainty about demand for fresh goods, the dynamic nature of traffic information, multiple distribution centers, multiple EV models, and time-of-use electricity prices. Thus, the following several aspects need to be addressed in future systematic in-depth studies: first, in the actual traffic environment, the EVRP problem should consider the dynamics and the speed randomness and study its specific representation. Second, further consideration should be given to different fresh goods damage and refrigeration temperature control. Third, more practical constraints can be introduced, e.g., conducting a comprehensive analysis of the urban cold chain distribution of the EVRP problem under different situations including multiple distribution centers, multiple vehicle types, and multimodal transport.

\section{Data Availability}

The data used to support the findings of this study are available from the corresponding author upon request.

\section{Conflicts of Interest}

The authors declare no conflicts of interest.

\section{Acknowledgments}

The authors would like to thank the great support by the National Natural Science Foundation of China (no. U1334207).

\section{References}

[1] C. Luo, Z. Shen, S. Evangelou, G. Xiong, and F.-Y. Wang, “The combination of two control strategies for series hybrid electric vehicles," IEEE/CAA Journal of Automatica Sinica, vol. 6, no. 2, pp. 596-608, 2019.

[2] B. A. Davis and M. A. Figliozzi, "A methodology to evaluate the competitiveness of electric delivery trucks," Transportation Research Part E: Logistics and Transportation Review, vol. 49, no. 1, pp. 8-23, 2013.

[3] W. Feng and M. Figliozzi, "An economic and technological analysis of the key factors affecting the competitiveness of electric commercial vehicles: a case study from the USA market," Transportation Research Part C: Emerging Technologies, vol. 26, pp. 135-145, 2013.

[4] Q. Kang, J. Wang, M. Zhou, and A. C. Ammari, "Centralized charging strategy and scheduling algorithm for electric vehicles under a battery swapping scenario," IEEE Transactions on Intelligent Transportation Systems, vol. 17, no. 3, pp. 659-669, 2016.

[5] B. Beltran, S. Carrese, E. Cipriani, and M. Petrelli, "Transit network design with allocation of green vehicles: a genetic algorithm approach," Transportation Research Part C: Emerging Technologies, vol. 17, no. 5, pp. 475-483, 2009.

[6] S. Erdoan and E. Miller-Hooks, "A green vehicle routing problem," Transportation Research Part E: Logistic and Transportation Review, vol. 48, no. 1, pp. 100-114, 2012.

[7] M. Schneider, A. Stenger, and D. Goeke, "The electric vehiclerouting problem with time windows and recharging stations," Transportation Science, vol. 48, no. 4, pp. 500-520, 2014.

[8] D. Goeke and M. Schneider, "Routing a mixed fleet of electric and conventional vehicles," European Journal of Operational Research, vol. 245, no. 1, pp. 81-99, 2015.

[9] H. Yang, S. Yang, Y. Xu, E. Cao, M. Lai, and Z. Dong, "Electric vehicle route optimization considering time-of-use electricity price by learnable partheno-genetic algorithm," IEEE Transactions on Smart Grid, vol. 6, no. 2, pp. 657-666, 2015.

[10] G. Desaulniers, F. Erric, S. Irnich et al., "Exact algorithms for electric vehicle-routing problems with time windows," Operations Research, vol. 64, no. 6, pp. 388-1405, 2016.

[11] W. C. Jie, J. Yang, and C. Yang, "Branch-and-price algorithm for heterogeneous electric vehicle routing problem," Systems Engineering-Theory \& Practice, vol. 36, no. 7, pp. 1795-1805, 2016.

[12] G. Hiermann, J. Puchinger, S. Ropke, and R. F. Hartl, “The electric fleet size and mix vehicle routing problem with time windows and recharging stations," European Journal of Operational Research, vol. 252, no. 3, pp. 995-1018, 2016.

[13] Y. Li, P. Zhang, and Y. Wu, "Public recharging infrastructure location strategy for promoting electric vehicles: a bi-level programming approach," Journal of Cleaner Production, vol. 172, pp. 2720-2734, 2018.

[14] A. Tiwari and P.-C. Chang, "A block recombination approach to solve green vehicle routing problem," International Journal of Production Economics, vol. 164, pp. 379-387, 2015.

[15] R. S. Kumar, K. Kondapaneni, V. Dixit, A. Goswami, L. S. Thakur, and M. K. Tiwari, "Multi-objective modeling of production and pollution routing problem with time window: a self-learning particle swarm optimization approach," Computers \& Industrial Engineering, vol. 99, pp. 29-40, 2016.

[16] M. Rabbani, A. Farshbaf-Geranmayeh, and N. Haghjoo, "Vehicle routing problem with considering multi-middle depots for perishable food delivery," Uncertain Supply Chain Management, vol. 4, pp. 171-182, 2016. 
[17] P. Amorim and B. Almada-Lobo, "The impact of food perishability issues in the vehicle routing problem," Computers \& Industrial Engineering, vol. 67, no. 1, pp. 223-233, 2014.

[18] Y. Wu, Z. J. Ma, and B. Zhen, "Integrated scheduling of production and distribution for perishable products with freshness requirements," Journal of Computer Applications, vol. 38, no. 4, pp. 1181-1188, 2018.

[19] W. F. Zhang and K. H. Liang, "Optimization of cold-chain network nodes and delivery for fresh agricultural products," System Engineering, vol. 35, no. 1, pp. 119-123, 2017.

[20] P. Devapriya, W. Ferrell, and N. Geismar, "Integrated production and distribution scheduling with a perishable product," European Journal of Operational Research, vol. 259, no. 3, pp. 906-916, 2017.

[21] Y. Li, F. Chu, C. Feng, C. Chu, and M. Zhou, "Integrated production inventory routing planning for intelligent food logistics systems," IEEE Transactions on Intelligent Transportation Systems, vol. 20, no. 3, pp. 867-878, 2019.

[22] A. E Ben, I. Hnrbnoui, P. Borne et al., "A comparative study of the PSO and GA for the m-MDPDPTW," International Journal of Computers Communications \& Control, vol. 13, no. 1, pp. 8-23, 2018.

[23] Z. Zhang, H. Qin, and Y. Li, "Multi-objective optimization for the vehicle routing problem with outsourcing and profit balancing," IEEE Transactions on Intelligent Transportation Systems, vol. 21, no. 5, pp. 1987-2001, 2020.

[24] H. Preis, S. Frank, and K. Nachtigall, "Energy-optimized routing of electric vehicles systems," in Operations Research Proceedings 2012, pp. 583-588, Springer, Cham, 2014.

[25] F. Angel, G. Righini, M. T. Ortuno et al., "A heuristic approach for the green vehicle routing problem with multiple technologies and partial recharges," Transportation Research Part E: Logistics and Transportation Review, vol. 71, pp. 111128, 2014.

[26] J. Bai, G.-K. Yang, Y.-W. Chen, L.-S. Hu, and C.-C. Pan, “A model induced max-min ant colony optimization for asymmetric traveling salesman problem," Applied Soft Computing, vol. 13, no. 3, pp. 1365-1375, 2013.

[27] L. Tang, J. He, K. Jie, and Z.-H. Jin, "Collaborative manufacturing scheduling based on improved ant colony optimization algorithm with time window constraint," Chinese Journal of Management Science, vol. 26, no. 4, pp. 97-107, 2018.

[28] M. Boukens, A. Boukabou, and M. Chadli, "A real time selftuning motion controller for mobile robot systems," IEEE/ CAA Journal of Automatica Sinica, vol. 6, no. 1, pp. 84-96, 2019.

[29] W. Luan, G. Liu, C. Jiang, and M. Zhou, "MPTR: a maximalmarginal-relevance-based personalized trip recommendation method," IEEE Transactions on Intelligent Transportation Systems, vol. 19, no. 11, pp. 3461-3474, 2018.

[30] S. Gao, M. Zhou, Y. Wang, J. Cheng, H. Yachi, and J. Wang, "Dendritic neuron model with effective learning algorithms for classification, Approximation, and prediction," IEEE Transactions on Neural Networks and Learning Systems, vol. 30, no. 2, pp. 601-614, 2019.

[31] H. Z. Zhang, Y. Liu, and J. Ni, "A partheno-genetic hybrid ant colony algorithm for solving the MOVRPFTW based on customer satisfaction," In Journal of Highway and Transportation Research and Development, vol. 28, no. 5, pp. 927933, 2019.

[32] J. Chen, P. F. Gui, T. Ding et al., "Optimization of transportation routing problem for fresh food by improved ant colony algorithm based on tabu search," Sustainability, vol. 11, no. 23, p. 6584, 2019.

[33] H. L. Tang, H. S. Tang, and X. L Zhu, "Research on low-carbon vehicle routing problem based on modified ant colony algorithm," Chinese Journal of Management Science, 2019.

[34] W. T. Fang and S. Z. Ai, "Research on cold chain logistics distribution path optimization based on hybrid ant colony algorithm," Chinese Journal of Management Science, vol. 27, no. 11, pp. 107-115, 2019.

[35] D. Zhang, J. C. Jiang, W. G. Zhang et al., "Optimal configuration of charging spots for electric Taxis," Transactions of China Electrotechnical Society, vol. 30, no. 18, pp. 181-188, 2015.

[36] The United States Advanced Battery Consortium (USABC), Electric Vehicle Battery Test Procedures, USABC, Southfield, MI, USA, 1996.

[37] Y. Xiao and A. Konak, "The heterogeneous green vehicle routing and scheduling problem with time-varying traffic congestion," Transportation Research Part E: Logistics and Transportation Review, vol. 88, pp. 146-166, 2016.

[38] C. S. Liu and X. C. Zhou, "Research on TDVRPTW of fresh E-commerce distribution: considering both economic cost and environmental cost," Control and Decision, vol. 35, no. 5, pp. 1273-1280, 2020.

[39] L. Li and T. J. Fan, "Comparison and analysis on pricing policies for fresh agricultural produce supply chain with dominant retailer," Chinese Journal of Management Science, vol. 23, no. 12, pp. 113-123, 2015.

[40] L. Li, S. X. Liu, and J. F. Tang, "Improved ant colony algorithm for solving vehicle routing problem with time windows," Control and Decision, vol. 25, no. 9, pp. 1379-1383, 2010.

[41] Q. Zhang and Y. Xiong, "Multi-objective model of distribution route problem for fresh electricity commerce under uncertain demand," Journal of System Simulation, vol. 9, pp. 1582-1590, 2019.

[42] M. Schiffer and G. Walther, "The electric location routing problem with time windows and partial recharging," European Journal of Operational Research, vol. 260, no. 3, pp. 995-1013, 2017.

[43] D. L. Li and Q. Cao, "Optimization of green fresh food logistics with heterogeneous fleet vehicle route problem by improved genetic algorithm," Sustainability, vol. 12, no. 5, 2020.

[44] J. Li and J. H. Zhang, "Vehicle routing problem with time windows based on carbon emissions and speed optimization," System Engineering Theory and Practice, vol. 34, no. 12, pp. 3063-3072, 2014. 